\title{
A note on higher-order vertices of higher-spin fields in flat and (A)dS space
}

\author{
Euihun Joung ${ }^{a}$ and Massimo Taronna ${ }^{b, c, d}$ \\ ${ }^{a}$ Department of Physics and Research Institute of Basic Science, Kyung Hee University, \\ Seoul 02447, Korea \\ ${ }^{b}$ Department of Physics, Princeton University, \\ Jadwin Hall, Princeton, NJ 08544, U.S.A. \\ ${ }^{c}$ Dipartimento di Fisica "Ettore Pancini", Università degli Studi di Napoli Federico II, \\ Monte S. Angelo, Via Cintia, 80126 Napoli, Italy \\ ${ }^{d}$ INFN, Sezione di Napoli, \\ Monte S. Angelo, Via Cintia, 80126 Napoli, Italy \\ E-mail: euihun.joung@khu.ac.kr, mtaronna@princeton.edu
}

ABSTRACT: In this work we classify (homogeneous) solutions to the Noether procedure in (A)dS for an arbitrary number of external legs and in general dimensions, analysing also the corresponding deformations of gauge symmetries. This builds upon the corresponding flat space classification [1], which we review and give its relation with the (A)dS result presented here. The role of dimensional dependent identities is studied in detail, which we find do not lead to new solutions for couplings involving more than three fields. For spins one and two our formalism recovers the Yang-Mills and Gravity examples.

Keywords: AdS-CFT Correspondence, Higher Spin Gravity, Higher Spin Symmetry, Scattering Amplitudes

ARXIV EPRINT: 1912.12357

Dedicated to the memory of our friend and colleague Dima Polyakov. 


\section{Contents}

1 Introduction 1

$\begin{array}{lll}1.1 & \text { Organisation of paper } & 7\end{array}$

2 Higher-order deformations \& Noether procedure $\quad 7$

3 Homogeneous solution in flat-space $\quad 8$

3.1 Homogeneous solution in flat-space: A review 8

$\begin{array}{lll}3.1 .1 & n=3 & 9\end{array}$

$\begin{array}{lll}3.1 .2 n=4 & 10\end{array}$

$\begin{array}{lll}3.1 .3 & \text { General } n & 13\end{array}$

$\begin{array}{lll}3.2 & \text { Comments on dimensional-dependent identities } & 14\end{array}$

3.3 Combining solutions: more than one massless field 20

4 Homogeneous solution in AdS 21

5 Particular solutions, current exchanges \& locality 23

$\begin{array}{ll}\text { A YM \& Gravity examples } & 28\end{array}$

\section{Introduction}

In this note we address the problem of classifying $n$-point couplings (where $n>3$ ) of massive and massless higher-spin fields in both flat space, where a classification was first given in $[1,2]$, and (A)dS space where a complete classification has not been yet available. ${ }^{1}$ This endeavour is part of the so called Noether procedure program [10] (see e.g. [10-20] for a few non-exhaustive list of references mostly about 3pt couplings $(n=3)$ and their classification). One of the main points of our approach is to reformulate the Noether program in a form in which locality is not manifest or built in, pursuing the idea of [1]. At the same time, one of the advantages of this approach is that it allows to have some quantitative control on the degree of non-locality of the most general Noether solution, as we shall demonstrate. More recently, this type of approach was taken in [21] which, along similar lines, ${ }^{2}$ led to the conclusion that HS theories in AdS present a degree of non-locality in the same functional class as exchange amplitudes, similar to their flat-space counterparts. This contradicts the initial expectations and raises an issue on the fieldtheoretical interpretation of (A)dS theories. It was thus suggested in [21] that reformulating

\footnotetext{
${ }^{1}$ Explicit results at quartic or higher orders are scarce, see e.g. [2-9] for various other efforts in flat space. ${ }^{2}$ See also [22-26] for other related results at the level of unfolded formulation [27].
} 
HS theories as topological string theories (see e.g. [28-32] for concrete proposals ${ }^{3}$ ) could be a way forward. Even though locality is a key ingredient of the Noether procedure for which so far no convincing replacement has been successfully proposed, we would like to remark here that it is still useful in principle to study the constraints of gauge invariance on $n$-point couplings in (A)dS regardless of their locality properties. Such tensorial structures indeed would still turn out to be holographic dual of corresponding $n$-point spinning structures which satisfy appropriate conservation conditions. ${ }^{4}$ Such structures are expected to play a role to describe stress-tensor and current correlators and the result presented in this paper would therefore find potentially interesting applications in this context. ${ }^{5}$

In the following, we first give a self-contained introduction of the main concepts of the Noether procedure program [10], for the sake of brevity leaving out most of the technical details which can be found in the literature (see the references given in the first paragraph of the introduction). We shall follow the presentation in $[2,20,46]$.

In the Noether procedure programme [10], the starting point is the expansion of the sought action and gauge transformations in powers of the fields:

$$
S=S^{(2)}+S^{(3)}+\cdots, \quad \delta_{\varepsilon} \varphi=\delta_{\varepsilon}^{(0)} \varphi+\delta_{\varepsilon}^{(1)} \varphi+\cdots .
$$

Here, the superscript $(n)$ means that the corresponding term involves $n$-th powers of the fields $\varphi$. In this weak-field expansion scheme, the gauge invariance of the action is recast into an infinite number of coupled equations:

$$
\delta_{\varepsilon} S=0 \Rightarrow\left\{\begin{array}{c}
\delta_{\varepsilon}^{(0)} S^{(2)}=0 \\
\delta_{\varepsilon}^{(0)} S^{(3)}+\delta_{\varepsilon}^{(1)} S^{(0)}=0 \\
\delta_{\varepsilon}^{(0)} S^{(4)}+\delta_{\varepsilon}^{(1)} S^{(3)}+\delta_{\varepsilon}^{(2)} S^{(2)}=0 \\
\vdots
\end{array}\right.
$$

where $S^{(2)}$ and $\delta_{\varepsilon}^{(0)} \varphi$ are the free action and the corresponding gauge transformations, respectively.

Higher order parts of the action, $S^{(n \geq 3)}$, and the gauge transformation, $\delta_{\varepsilon}^{(n \geq 1)} \varphi$, can be identified starting from $S^{(2)}$ and $\delta_{\varepsilon}^{(0)} \varphi$ by solving the above equations. The strategy is to solve the equations (i) of (1.2) in two steps as

$$
S^{(2)}, \delta_{\varepsilon}^{(0)} \varphi \stackrel{(1)}{\longrightarrow} S^{(3)} \stackrel{(1)}{\longrightarrow} \delta_{\varepsilon}^{(1)} \varphi \stackrel{(2)}{\longrightarrow} S^{(4)} \stackrel{(2)}{\longrightarrow} \delta_{\varepsilon}^{(2)} \varphi \longrightarrow \cdots
$$

\footnotetext{
${ }^{3}$ Although the formulation of HS theories as field theories in AdS is currently under debate, it is still possible in principle to formulate a Frobenious-Chern Simons theory on non-commutative 10d space based on the higher-spin algebra [31]. The precise relation between such approach and a stringy-AdS/CFT picture is still an open question which in order to be settled would require to properly define admissible boundary conditions in the whole non-commutative space. A first proposal in this direction have been set forth and tested to the linearised level in [33, 34]. It would be important to clarify whether such proposal is valid at the fully non-linear level. In any case, classifying admissible boundary conditions of the Frobenious Chern Simons theory on non-commutative space is an interesting mathematical question which might ultimately shed light on how to relate the Frobenious Chern Simons formulation to a more standard stringy/holographic formulation of higher-spin theories at the interacting level.

${ }^{4}$ For $n=3$ this map was written down in [35] for arbitrary integer spinning legs.

${ }^{5}$ Note also that if one works in 3d without matter [36-38], or gives up the assumptions of unitarity [39-41] or allows complex actions [42-44] and [45] one can find examples of higher-spin theory actions.
} 
where (n) represents the same condition (n) but solved on the shell of free EoM. In particular, at each order one can first solve for $S^{(n+2)}$ using (n) and then read off $\delta_{\varepsilon}^{(n)} \varphi$ from (n). On the other hand, the full non-linear gauge transformations must form an (open) algebra:

$$
\delta_{\varepsilon_{1}} \delta_{\varepsilon_{2}} \varphi-\delta_{\varepsilon_{2}} \delta_{\varepsilon_{1}} \varphi \approx \delta_{\llbracket \varepsilon_{1}, \varepsilon_{2} \rrbracket} \varphi
$$

where the commutator $\llbracket \varepsilon_{1}, \varepsilon_{2} \rrbracket$ is in principle field-dependent and can be expanded as

$$
\llbracket \varepsilon_{1}, \varepsilon_{2} \rrbracket=\llbracket \varepsilon_{1}, \varepsilon_{2} \rrbracket^{(0)}+\llbracket \varepsilon_{1}, \varepsilon_{2} \rrbracket^{(1)}+\cdots .
$$

For the purpose of the present note, one first focuses on the lowest-order part of the commutator:

$$
\delta_{\varepsilon_{1}}^{(0)} \delta_{\varepsilon_{2}}^{(1)} \varphi-\delta_{\varepsilon_{2}}^{(0)} \delta_{\varepsilon_{1}}^{(1)} \varphi=\delta_{\llbracket \varepsilon_{1}, \varepsilon_{2} \rrbracket}^{(0)} \varphi
$$

which is field-independent and can be entirely obtained from $\delta_{\varepsilon}^{(1)}$, which was carried out explicitly in [46]. To sum up, once consistent cubic interactions are determined for a given free theory, one can the study the induced deformations of the gauge transformations and also of the gauge algebra:

$$
S^{(3)} \Rightarrow \delta_{\varepsilon}^{(1)} \varphi \quad \Rightarrow \quad \llbracket \varepsilon_{1}, \varepsilon_{2} \rrbracket^{(0)} .
$$

Closure of the higher-spin transformations then uniquely identifies the possible higherspin algebras [47], which in turn fixes all coupling constants at cubic order [48]. In this sense the free Fronsdal action contains all the information about the deformation of the gauge symmetries of the theory and their representations which together with closure fully specifies the higher-spin algebra structure constants. In the following, we shall consider the constraints which a massless, bosonic, symmetric fields imposes on the homogeneous solutions in both flat and (A)dS space. Including more than one massless field is equivalent to consider the intersection of the corresponding solution spaces for a single massless leg. In this way we will be able to classify the corresponding solutions with any number of massless and massive legs.

Ambient-space formulation and transverse and traceless part. In order to treat fields in (A)dS in a simple manner, we introduce the ambient-space formulation where fields $\varphi_{\mu_{1} \cdots \mu_{s}}(x)$ are described through the corresponding ambient avatars $\Phi_{M_{1} \cdots M_{s}}(X)$, defined in a $(d+1)$-dimensional flat space and subject to homogeneity and tangentiality conditions:

$$
\left(X \cdot \partial_{X}-U \cdot \partial_{U}+2-\mu\right) \Phi(X, U)=0, \quad X \cdot \partial_{U} \Phi(X, U)=0 .
$$

The mass of the field is here parametrised by the degree of homogeneity $\mu$, and when the field is massless (that is $\mu=0$ ), it admits gauge symmetries:

$$
\delta_{E}^{(0)} \Phi=U \cdot \partial_{X} E \quad\left[\partial_{U}^{2} E=0,\left(X \cdot \partial_{X}-U \cdot \partial_{U}\right) E=0, X \cdot \partial_{U} E=0\right] .
$$

In the following we shall work with ambient space fields satisfying the latter homogeneity conditions. These homogeneity conditions should be contrasted with those used in [35, 49, 50] which are more directly tuned to AdS/CFT calculations. The dictionary between 
the two formulation is however straightforward as discussed in the latter references. In constructing gauge-invariant interaction vertices, we focus for simplicity on the transverse and traceless (TT) part of the latter disregarding the terms proportional to divergences and traces of the fields. This is equivalent to consider, instead of the full vertices and their gauge variations, their quotient modulo the following equivalence relations:

$$
\partial_{U} \cdot \partial_{X} \Phi \stackrel{\text { TT }}{=} 0, \quad \partial_{U}^{2} \Phi \stackrel{\text { TT }}{=} 0 ; \quad \partial_{U} \cdot \partial_{X} E \stackrel{\text { TT }}{=} 0, \quad \partial_{X}^{2} E \stackrel{\text { TT }}{=} 0 .
$$

In this setting, the free action assumes a general form and is given simply by

$$
S^{(2)}[\Phi] \stackrel{\mathrm{TT}}{=}-\left.\frac{1}{2} \int_{(\mathrm{A}) \mathrm{d} S} e^{\partial_{U_{1}} \cdot \partial_{U_{2}}} \Phi\left(X, U_{1}\right) \partial_{X}^{2} \Phi\left(X, U_{2}\right)\right|_{U_{i}=0} .
$$

Within this description one can also describe the interacting part of action $S^{(n \geq 3)}[\Phi]$ together with the corresponding gauge transformations $\delta_{E}^{(n \geq 1)} \Phi$. For instance, the interaction parts of the action can be conveniently expressed as

$$
\left.S^{(n)}[\Phi] \stackrel{\mathrm{TT}}{=} \int_{(\mathrm{A}) \mathrm{d} S} C^{(n)} \Phi\left(X_{1}, U_{1}\right) \cdots \Phi\left(X_{n}, U_{n}\right)\right|_{\substack{X_{i}=X \\ U_{i}=0}},
$$

in terms of a differential operator $C^{(n)}$ in $X_{i}$ and $U_{i}$. Each operator $C^{(n)}$ is constrained by the gauge-invariance conditions (1.2).

$\boldsymbol{H}$ vs $\boldsymbol{G}$ structures. An important lesson that was first drawn in $[46,51,52]$ is that cubic interactions corresponding to trivial deformations of the gauge symmetries are related to the existence of a tensor structure $H_{i j}$ :

$$
H_{i j}=\partial_{U_{i}} \cdot \partial_{X_{j}} \partial_{U_{j}} \cdot \partial_{X_{i}}-\partial_{X_{i}} \cdot \partial_{X_{j}} \partial_{U_{i}} \cdot \partial_{U_{j}} \cdot
$$

The $H_{i j}$ 's are operators taking the ambient space curls of the $i$-th and $j$-th fields and contracting them. They are gauge invariant without making use of the on-shell condition, and hence, they do not lead to any deformation of the gauge transformations.

The above $H$-structures should be confronted with the actual classification of massless interactions which can be expressed in terms of what was referred to as $G$-structure in [18]:

$$
G=\partial_{U_{1}} \cdot \partial_{X_{2}} \partial_{U_{2}} \cdot \partial_{U_{3}}+\text { cyclic, }
$$

and are in particular proportional to powers $G^{n}$ of the YM structure $G$ up to appropriately tuned lower derivative terms (see e.g. eq. (2.4) of [46]). In particular, in [19] the above contractions of $\partial_{U}$ and $\partial_{X}$ used to define cubic couplings via a generating function notation were defined as

$$
Y_{i}=\partial_{U_{i}} \cdot \partial_{X_{i+1}}, \quad Z_{i}=\partial_{U_{i-1}} \cdot \partial_{U_{i+1}}
$$

with $i \sim i+3$. Therefore, the classification of gauge deformations boils down to the question whether a $G$-coupling of the form:

$$
C^{(3)} \sim Y_{1}^{s_{1}-k} Y_{2}^{s_{2}-k} Y_{3}^{s_{3}-k} G^{k}+\mathcal{O}(\Lambda),
$$


A

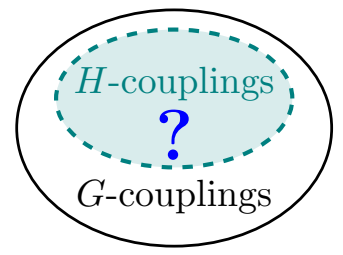

B

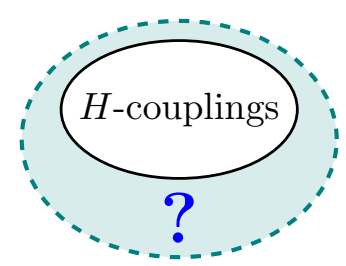

Figure 1. Schematic diagrammatic representation of gauge invariant couplings. The smaller subset represent those couplings which up to a field redefinition are gauge invariant off-shell. The bigger set represent instead the space of couplings whose gauge variation is proportional to the EoMs. It is important to note that there might in principle exist couplings which are not proportional to the EoMs and whose gauge transformation instead is.

is expressible (up to field redefinitions) as a $H$-coupling namely a function of the $H_{i j}$ structures (1.13) which are off-shell gauge invariant: see e.g. the diagram $\mathbf{A}$ of figure 1. On the other hand, one can also view this problem in the opposite perspective, as the question whether there are additional couplings besides those which are trivially gauge-invariant and expressed in terms of the $H$-structures: see the diagram $\mathbf{B}$ of figure 1 . The latter viewpoint is natural in the sense that $H$-couplings always provide consistent interactions while $G$-couplings are gauge-invariant on-shell and therefore arise only in some special cases as, for instance, the case of three-massless-field interactions. This question was addressed in great detail in $[51,52]$, where it was shown that also in more general classes of gauge interactions (e.g. involving partially-massless or massive fields), $G$-couplings arises only when the field masses satisfy specific conditions whereas $H$-couplings exist always and are furthermore manifestly gauge-invariant off-shell. In this note we shall demonstrate how this simple picture turns out to be applicable also beyond the cubic order and extends to higher-order interactions in a suggestive way.

Summary of higher-order results. In order to extend the cubic analysis to higher orders, following [1], the main idea is to split the interaction term into two parts:

$$
C^{(n)}=C_{p}^{(n)}+C_{h}^{(n)} .
$$

Here $C_{p}^{(n)}$ is the particular-solution to (n) in the sense that it deals with the compatibility with the lower $n$ interactions $C_{p}^{(m<n)}$, and $C_{h}^{(n)}$ is any homogeneous-solution part solving $\delta_{\varepsilon}^{(0)} S_{h}^{(n)}+\delta_{\varepsilon}^{(n)} S^{(2)}=0$ and talking (a priori) with the quadratic action only. The main point is that, although both $C_{p}^{(n)}$ and $C_{h}^{(n)}$ taken by themselves are generically non-local objects, they admit a simple physical interpretation which allows to find a formal solution to the Noether procedure to any order in a weak field expansion [1,21]. Then, given a certain particular solution $C_{p}^{(n)}$ which we shall discuss in section 5 , one can add any homogeneous solution $C_{h}^{(n)}$. Locality is reformulated in this language as the condition that all non-localities in $C_{p}^{(n)}$ are compensated by a certain choice of $C_{h}^{(n)}$ (if this choice exists). This condition, when a solution exists, recursively relates higher-order contact interactions to lower ones reconstructing a full non-linear theory.

The general solution for $C_{h}^{(n)}$ can now be obtained by requiring its on-shell gauge invariance w.r.t. the free EoM. Hence, this corresponds to a straightforward generalisation 


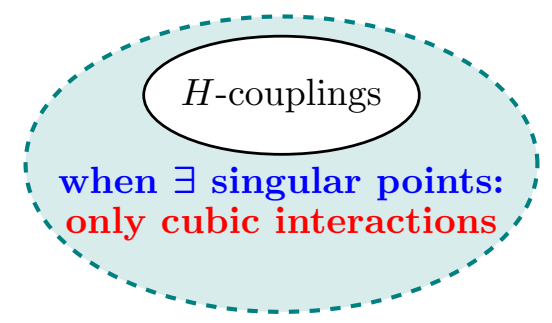

Figure 2. Schematic diagram of the coupling space.

of the cubic-interaction problem to higher orders. ${ }^{6}$ Its general solution will be shown in this note to be given by

$$
C_{h}^{(n)}=K^{(n)}\left(W^{i j}, H_{i j}, H_{i}^{j k}\right), \quad[n \geq 4],
$$

where we also introduced the structure $W^{i j}=\partial_{X_{i}} \cdot \partial_{X_{j}}$, which is non-trivial for $n>3$, the $H_{i j}$ 's are given in (1.13) while $H_{i}^{j k}$ 's are:

$$
H_{i}^{j k}=\partial_{X_{i}} \cdot \partial_{X_{j}} \partial_{X_{k}} \cdot \partial_{U_{i}}-\partial_{X_{i}} \cdot \partial_{X_{k}} \partial_{X_{j}} \cdot \partial_{U_{i}} \cdot
$$

The $H$ structures above are nothing but the generalisations of the cubic $H$ structures to the case in which more external legs are present and have the same defining curl-structure!

It is interesting to stress here that we can prove no $G$ type solution can be constructed for $n>3$ and that, although we were searching in general for on-shell gauge invariant structures, all the above solutions are gauge invariant without relying on the on-shell condition in the same way as the $H_{i j}$ structure in (1.13).

This observation, which was made in [7], implies that $C_{h}^{(n)}$ does not induce any nontrivial deformation of the gauge transformations and that the only deformations of gauge transformations are induced by $3 p t$ structure glued together with homogeneous solutions in constructing more general particular solutions, more details on this construction will be given in section 5 .

Eventually, the seed of the only non-trivial deformations of gauge transformations arises from the particular solution $C_{p}^{(n)}$ that in turn can be recursively related to the cubic couplings glued together with other homogeneous solutions.

The fact that the only homogeneous solutions which are associated with non-trivial deformations of the gauge transformations are cubic couplings can also be rephrased in the language of differential equations. Indeed the $G$-solutions discussed around (1.14) appear only when differential equations which we derive from the gauge invariance condition admit some singular points. ${ }^{7}$ At cubic order such singular points exist in the case of

${ }^{6}$ This problem is equivalent to identifying all deformations that start at the $n$-th order in the fields:

$$
S=S^{(2)}+S^{(n)}+S^{(n+1)}+\cdots .
$$

\footnotetext{
${ }^{7} \mathrm{~A}$ singular point of a partial differential equation is a point at which some of the equation coefficient have a singularity.
} 
three-massless-field interactions and the interactions among one massless and two massive fields with special mass differences [51, 52]. On the other hand, for the other cases of three-field interactions and also higher-order interactions, this never happens since the field masses (cubic case) or Mandelstam variables prevent the coefficients of the PDE to vanish identically.

\section{$1.1 \quad$ Organisation of paper}

The paper is organised as follows. In section 2 we briefly review the structure of Noether procedure equations. In section 3 we discuss in detail the case of flat space interactions, reviewing relevant exisiting results. In section 3.2 we argue that for $n>3$ DDIs do not change the solution space. In section 4 we extend the analysis to constant curvature background presenting the corresponding solution. We conclude in section 5 with a discussion of the role of the particular solution as for regards deformations of gauge transformations and with further concluding remarks. We relegate some technical details and the examples of YM and Gravity to the appendices A.

We became aware of a flat space analysis by Stefan Fredenhagen, Olaf Krüger and Karapet Mkrtchyan with potential overlap with the one [1,2] we review in this work and with our new results on dimensional dependent identities. We have arranged to synchronise the submission of our papers.

\section{Higher-order deformations \& Noether procedure}

To begin with, let us consider the $n$-th order part of the gauge invariance condition of the sought interacting action:

$$
\delta^{(0)} S^{(n)}+\delta^{(n-2)} S^{(2)}=-\left(\delta^{(1)} S^{(n-1)}+\cdots+\delta^{(n-3)} S^{(3)}\right),
$$

where we assume the interaction terms $S^{(2)}, \ldots, S^{(n-1)}$ to be already determined. In order to solve the above equation, we again consider first the on-shell version:

$$
\delta^{(0)} S^{(n)} \approx-\left(\delta^{(1)} S^{(n-1)}+\cdots+\delta^{(n-3)} S^{(3)}\right),
$$

whose general solution $S^{(n)}$ is the sum of the particular solution $S_{p}^{(n)}$ and homogeneous solution $S_{h}^{(n)}$ :

$$
S^{(n)}=S_{p}^{(n)}+S_{h}^{(n)}, \quad\left\{\begin{array}{c}
\delta^{(0)} S_{p}^{(n)} \approx-\left(\delta^{(1)} S^{(n-1)}+\cdots+\delta^{(n-3)} S^{(3)}\right) . \\
\delta^{(0)} S_{h}^{(n)} \approx 0
\end{array} .\right.
$$

Note that the particular solution is defined starting from $n=4$ and so on. In the following, we will find the solutions of the above equations and study their implications in an analogous manner to the cubic-interaction analysis, extending the analysis of [1] to (A)dS and the analysis of [19] to higher-order couplings.

It is interesting to note on a side that the homogeneous solution $S_{h}^{(n)}$ also plays the role of first non-trivial coupling in a theory with no lower-point interactions: ${ }^{8}$

$$
S=S^{(2)}+S^{(n)}+S^{(2 n-2)}+\ldots
$$

\footnotetext{
${ }^{8}$ See $[15,16,53]$ for some discussions on local higher-order deformations for $n>3$ and spin $s \leq 4$.
} 
We will see how our analysis will show that cubic interaction have a special role in determining non-trivial deformations of the theory and that theories with no cubic couplings would always give rise to trivial deformations of the gauge symmetries of the free theory. ${ }^{9}$

\section{Homogeneous solution in flat-space}

In section 3.1 we review some flat space results about the Noether procedure for higher-spin fields beyond the cubic-order and in general dimension [1, 2, 7], giving additional details which are relevant for the (A)dS generalisation. In particular, the generating-functions for all homogeneous solutions in $d$-dimensional flat space was first given in [1], which can be found in the latter reference under equation (4.41) for quartic interactions and equation (A.2) for $n$-point interactions. These results were expressed as polynomials of the gauge boson and scalar structures, defined in equation (4.27) of that reference. These results were then given in equation (28) of [2] using a different basis of structures from (4.27) in [1], which are more suitable for the AdS generalisation. For this reason, our analysis is closer to that outlined in the work [2] and the expressions we give in equations (3.32) and (4.12) for all $n$-point homogenous solutions in flat and AdS space are identical to equation (28) of [2], where this form for the solution was first given in the literature 8 years ago.

In section 3.2 we show that taking into account dimensional dependent identities does not change the classification, which is a new result.

\subsection{Homogeneous solution in flat-space: A review}

First of all it is convenient to work with the generating function formalism:

$$
\phi(x, u)=\frac{1}{\ell !} \phi_{\mu_{1} \ldots \mu_{\ell}}(x) u^{\mu_{1}} \cdots u^{\mu_{\ell}} .
$$

One can then express a generic homogeneous solution as a function of certain basic tensor contractions which are in one to one correspondence with the possible contractions of indices of the tensors $\phi_{\mu_{1} \ldots \mu_{\ell}}(x)$. Assuming parity invariance and working in generic dimension the most general TT part for a given $n$-point homogeneous solution in flat space can be therefore written as

$$
S_{h}^{(n)}=\int_{\mathbb{R}^{d}} C_{h}^{(n)}\left(w^{i j}, y_{i}^{j}, z_{i j}\right) \prod_{i=1}^{n} \phi\left(x_{i}, u_{i}\right),
$$

where we have defined the basic contractions

$$
w^{i j}=\partial_{x_{i}} \cdot \partial_{x_{j}}, \quad y_{i}^{j}=\partial_{u_{i}} \cdot \partial_{x_{j}}, \quad z_{i j}=\partial_{u_{i}} \cdot \partial_{u_{j}} .
$$

with $i \neq j$ since $i=j$ terms are removed by the TT conditions or are proportional to the mass-shell condition. Counting the number of structures one easily gets

$$
(\# \text { of } w)=\frac{1}{2} n(n-3), \quad(\# \text { of } y)=n(n-2), \quad(\# \text { of } z)=\frac{1}{2} n(n-1) .
$$

\footnotetext{
${ }^{9}$ It is important to note that constructing Abelian theories of HS fields in both AdS and flat space is not a difficult task (see e.g. [54]). What makes the HS problem non-trivial is the requirement of minimal coupling to gravity which requires a very special set of $3 \mathrm{pt}$ couplings to be turned on.
} 
Each local coupling would then be encoded into a polynomial in the above variables. The variables $w^{i j}$, up to a Fourier transform, play the role of generalised Mandelstam invariants and become non-trivial only for $n \geq 4$. The condition of gauge invariance can be formulated on the full space spanned by the variables (3.3) and takes the form of the following linear PDE:

$$
\left[C^{(n)}(s, y, z), u_{1} \cdot \partial_{x_{1}}\right] \approx\left(y_{i}^{1} \partial_{z_{i 1}}+w^{1 j} \partial_{y_{1}^{j}}\right) C_{h}^{(n)}(w, y, z) \sim 0
$$

where in this equation we use Einstein summation convention for repeated indices. In the formulation above the symbol $\sim$ implies that the above equation should be solved on the space of formal polynomials subject to the following equivalence relations:

$$
\chi_{1}^{i}(w, y, z)=\sum_{j} w^{i j} \sim 0, \quad \chi_{2}^{i}(w, y, z)=\sum_{j} y_{i}^{j} \sim 0 .
$$

This simply encodes the fact that gauge invariance should hold in the most general case up to integrations by part and boundary terms. On top of this we should also allow for couplings which become gauge invariant on the linear mass-shell and in our setting we also quotient by the TT conditions:

$$
\chi_{3}^{i}(w, y, z)=w^{i i} \sim 0, \quad \chi_{4}^{i}(w, y, z)=y_{i}^{i} \sim 0, \quad \chi_{5}^{i}(w, y, z)=z_{i i} \sim 0 .
$$

In practise the problem can be reformulated as the following inhomogeneous differential equation:

$$
\left(y_{i}^{1} \partial_{z_{i 1}}+w^{1 j} \partial_{y_{1}^{j}}\right) C_{h}^{(n)}(w, y, z)=\sum_{a=1}^{5} \sum_{i=1}^{n} \chi_{k}^{i}(w, y, z) f_{a}^{(i)}(w, y, z),
$$

for some generic polynomial functions $f_{a}^{(i)}$ which implement the equivalence relations (3.6) and (3.7). Therefore, the general structure of the solution space to the above problem is given by the generic solution to the homogeneous equation which turns out to have a generic form for arbitrary $n$ to which we shall add all possible particular solutions available when varying the functions $f_{a}^{(i)}$ in the space of polynomials.

One way to address and solve the above set of equations is to pick a representative for the equivalence relation (3.6) and (3.7). The case $n=3$ is well known and was discussed in $[17,18]$. In the following we shall briefly review the $n=3$ case, we shall then address the $n=4$ example in detail and then review the extension of the analysis to $n \geq 4[1,2,7]$.

\subsection{1 $n=3$}

In the $n=3$ case it is easy to solve the relation (3.6) and (3.7) in terms of the following subset of independent variables:

$$
\Xi=\left\{y_{1}^{2}, y_{2}^{3}, y_{3}^{1}, z_{12}, z_{23}, z_{31}\right\}
$$

where we note that $w_{i j} \approx 0$ for all $i$ and $j$. The differential equation (3.5) then becomes [19]

$$
\left(y_{2}^{1} \partial_{z_{12}}-y_{3}^{1} \partial_{z_{13}}\right) C_{h}^{(3)}(y, z)=0 .
$$


The characteristics of the above equation then satisfy

$$
\frac{d y_{i}^{j}}{d \sigma}=0, \quad \frac{d z_{12}}{d \sigma}=y_{2}^{1}, \quad \frac{d z_{13}}{d \sigma}=-y_{3}^{1}, \quad \frac{d z_{23}}{d \sigma}=0,
$$

and the solutions can be obtained by integration as

$$
z_{12}=y_{2}^{1} \sigma+c_{12}, \quad z_{13}=-y_{3}^{1} \sigma+c_{13} .
$$

Since $\frac{d C}{d \sigma}=0$, the solution of (3.11) is a function of $\sigma$-constants. Besides the other trivially constant variables in $\sigma$, one recovers an additional $\sigma$-constant,

$$
g=z_{12} y_{3}^{1}+z_{13} y_{2}^{1},
$$

by eliminating the $\sigma$ dependence from (3.12). In the end, we obtained the general $3 \mathrm{pt}$ solution with one massless field as [51]

$$
C_{h}^{(3)}=\mathcal{K}^{(3)}\left(g, z_{23}, y_{1}^{2}, y_{2}^{3}, y_{3}^{1}\right) \text {. }
$$

\subsection{2 $n=4$}

The $n=4$ example differs from the $n=3$ example from the fact that it is possible to define non-trivial Mandelstam invariants. For this reason this case will show the main features which will hold also for higher points and provides us with the main intuition which we shall generalise in the following.

The first step is to solve the equivalence relation in terms of a set of independent variables:

$$
\Xi=\left\{w^{12}, w^{14}, y_{1}^{2}, y_{1}^{4}, z_{12}, z_{13}, z_{14}, \text { (cyclic permutations) }\right\},
$$

among which the variables relevant to the gauge variation of the point-splitted field $\phi_{1}$ are

$$
y_{1}^{2}, \quad y_{1}^{4} \quad z_{12}, \quad z_{13}, \quad z_{14}
$$

whereas the rest of variables can be considered as constants.

The gauge invariance equation then reads

$$
\left(w^{12} \partial_{y_{1}^{2}}+w^{14} \partial_{y_{1}^{4}}+y_{2}^{1} \partial_{z_{12}}-\left(y_{3}^{2}+y_{3}^{4}\right) \partial_{z_{13}}+y_{4}^{1} \partial_{z_{14}}\right) C^{(4)}(w, y, z)=0 .
$$

Note that the variables $y_{2}^{1}, y_{3}^{4}, y_{3}^{2}, y_{4}^{1}, w^{12}$ and $w^{14}$, besides the ones in (3.16), do appear in the equation, but they can still be considered as constants since there is no differentiation with respect to them. The characteristics of (3.17) satisfy

$$
\begin{aligned}
& \frac{d y_{1}^{2}}{d \sigma}=w^{12}, \quad \frac{d y_{1}^{4}}{d \sigma}=w^{14}, \\
& \frac{d z_{12}}{d \sigma}=y_{2}^{1}, \quad \frac{d z_{14}}{d \sigma}=y_{4}^{1}, \quad \frac{d z_{13}}{d \sigma}=-\left(y_{3}^{2}+y_{3}^{4}\right) \equiv y_{3}^{1}(\Xi),
\end{aligned}
$$

while all the other variables are constant in $\sigma$. The above system has an analogous structure as for 3pt: the derivatives of the $z$ variables are expressed in terms of linear combinations of $y_{i}^{1}$ and the derivatives of $y_{1}^{i}$ are expressed in terms of linear combinations of $w^{1 i}$ variables. 
Since the right hand sides of the equations, namely the inhomogeneous part of the ODEs are constants, the solutions to (3.19) are linear functions of $\sigma$.

In conclusions, since all characteristics in (3.16) are linear functions of $\sigma$, we can make a number of constant functions by linearly combining two of the characteristics in all possible ways and eliminating the $\sigma$ dependence. First, by taking linear combination of two $z_{1 i}$ 's with constant coefficients $y_{j}^{1}$, we get

$$
\begin{aligned}
& h_{24}=z_{12} y_{4}^{1}-z_{14} y_{2}^{1}, \\
& h_{23}=z_{12} y_{3}^{1}-z_{13} y_{2}^{1}, \\
& h_{34}=z_{13} y_{4}^{1}-z_{14} y_{3}^{1} .
\end{aligned}
$$

Note in $h_{34}$ we have left implicit the dependence of $y_{3}^{1}$ on the actual independent set of variables $\Xi$ for the sake of simplicity. Second, by linearly combining one $y_{1}^{i}$ and one $z_{1 j}$ with coefficients $y_{j}^{1}$ and $w^{1 i}$, we get

$$
\begin{aligned}
& h_{2}^{2}=z_{12} w^{12}-y_{1}^{2} y_{2}^{1}, \\
& h_{2}^{4}=z_{12} w^{14}-y_{1}^{4} y_{2}^{1}, \\
& h_{4}^{2}=z_{14} w^{12}-y_{1}^{2} y_{4}^{1}, \\
& h_{4}^{4}=z_{14} w^{14}-y_{1}^{4} y_{4}^{1}, \\
& h_{3}^{2}=z_{13} w^{12}-y_{1}^{2} y_{3}^{1}, \\
& h_{3}^{4}=z_{13} w^{14}-y_{1}^{4} y_{3}^{1} .
\end{aligned}
$$

Finally, by taking linear combination of two $y_{1}^{i}$ 's with coefficients $w^{1 j}$, we get

$$
h^{24}=y_{1}^{2} w^{14}-y_{1}^{4} w^{12} .
$$

The general solution of (3.17) is then given by any polynomial function

$$
C^{(4)}=\mathcal{K}^{(4)}\left(h_{i j}, h_{i}^{j}, h^{i j}, c_{a}\right),
$$

where $c_{a}$ are all the constant characteristics and $h_{i j}, h_{j}^{i}$ and $h^{i j}$ are

$$
\begin{aligned}
h_{i j} & =z_{1 i} y_{j}^{1}-z_{1 j} y_{i}^{1}, \\
h_{i}^{j} & =z_{1 i} w^{1 j}-y_{1}^{j} y_{i}^{1}, \\
h^{i j} & =y_{1}^{i} w^{1 j}-y_{1}^{j} w^{1 i} .
\end{aligned}
$$

Note that comparing the above to the lists in (3.20), (3.21) and (3.22), we find additional structures but they are in fact related to the others by

$$
h_{2}^{3}=-h_{2}^{2}-h_{2}^{4}, \quad h_{3}^{3}=-h_{3}^{2}-h_{3}^{4}, \quad h_{4}^{3}=-h_{4}^{2}-h_{4}^{4}, \quad h^{32}=-h^{34}=h^{24} .
$$

Therefore, we see that all non-trivial solutions of (3.17) are given by the $h$-structures (3.24) up to the above redundancies. 
A general consequence of the above finding is that all gauge invariant 4pt interactions do not induce a deformation of gauge symmetry. To see this point, it is enough to realise that the $h$-structures (3.24) are all based on curl-type operations:

$$
\begin{aligned}
h_{i j} & =2\left(\partial_{u_{i}}\right)_{\mu}\left(\partial_{u_{j}}\right)_{\nu}\left(\partial_{u_{1}}\right)^{[\mu}\left(\partial_{x_{1}}\right)^{\nu]}, \\
h_{i}^{j} & =2\left(\partial_{u_{i}}\right)_{\mu}\left(\partial_{x_{j}}\right)_{\nu}\left(\partial_{u_{1}}\right)^{[\mu}\left(\partial_{x_{1}}\right)^{\nu]}, \\
h^{i j} & =2\left(\partial_{x_{i}}\right)_{\mu}\left(\partial_{x_{j}}\right)_{\nu}\left(\partial_{u_{1}}\right)^{[\mu}\left(\partial_{x_{1}}\right)^{\nu]},
\end{aligned}
$$

where $T^{[\mu \nu]}=\frac{1}{2}\left(T^{\mu \nu}-T^{\nu \mu}\right)$. This makes gauge invariance manifest even without imposing the equivalence relations (3.6) and (3.7) simply because

$$
\left[\left(\partial_{u_{1}}\right)^{[\mu}\left(\partial_{x_{1}}\right)^{\nu]}, u_{1} \cdot \partial_{x_{1}}\right]=0 .
$$

It is interesting to also compare the $n=4$ result with the result obtained at $3 p t$. The basic structure of the solution is very similar and the $h$-structures we obtained at $n=4$ play the role of straightforward generalisations of the $g$ structure (3.13). However the main difference is that while the $y_{1}^{2}$ structures was associated with a constant characteristic in $\sigma$ for $n=3$, the $y_{1}^{j}$ structures are not anymore constant for $n=4$ but they can only appear in the combination given by the $h$-structures (3.21). This implies a key difference between $n=3$ and $n=4:$ All solutions to the gauge invariance conditions are gauge invariant off-shell, without using neither the TT or the mass-shell condition. We therefore recover as a corollary that no non-trivial deformation of the gauge symmetries of the free theory can be induced by an $n$-point coupling which solves the homogeneous equation $\delta^{(0)} S_{h}^{(n)} \approx 0 .{ }^{10}$

Let us reiterate the difference between $3 p t$ and $4 \mathrm{pt}$ couplings with the example of the interactions involving only one massless field. The PDE for the cubic interaction reads

$$
\left[y_{2}^{1} \partial_{z_{21}}-y_{3}^{2} \partial_{z_{31}}+\left(m_{2}^{2}-m_{3}^{2}\right) \partial_{z_{1}^{3}}\right] C^{(3)}=0
$$

displaying a singular point at $m_{2}^{2}-m_{3}^{2}=0$. On the other hand the PDE for quartic coupling is given by the following expression

$$
\left[y_{2}^{1} \partial_{z_{21}}+y_{3}^{1} \partial_{z_{31}}-\left(y_{4}^{2}+y_{4}^{3}\right) \partial_{z_{41}}-t \partial_{y_{1}^{3}}-\frac{1}{2}\left(s-m_{2}^{2}-m_{3}^{2}-m_{4}^{2}\right) \partial_{y_{1}^{4}}\right] C_{h}^{(4)}=0 .
$$

Here, the appearance of the Mandelstam variables for the first time at $n=4$ allows to avoid any singular point, the latter becoming of measure zero. One can summarise the above facts considering the generic form of the gauge-consistency PDE:

$$
\left(y_{a} \mathbb{A}^{a b} \partial_{z_{b}}-\mathbb{B}^{c} \partial_{y_{c}}\right) C=0
$$

where depending on the structure of the coefficient $\mathbb{B}$ one has two cases:

- $\mathbb{B}=0$ : Singular points of DE $\Rightarrow$ Both on/off-shell invariant couplings

- $\mathbb{B} \neq 0$ : No singular point $\Rightarrow$ Only off-shell gauge invariant couplings

\footnotetext{
${ }^{10}$ See [7] (and [15, 16, 53] up to spin $\left.s \leq 4\right)$ for previous works where similar statements were made.
} 
All in all, the presence of Mandelstam variables within the coefficient $\mathbb{B}$ starting at $n=4$ implies that the PDE does not have any singular point. Hence, this excludes the possibility of having further couplings with respect to the ones described above and proves also the non-deforming nature of the homogeneous solutions for $n=4$. The only homogeneous solutions that can be non-Abelian arise at the cubic level due to the non-trivial singular points of the corresponding gauge-invariance PDE.

In the following we shall review the generalisation of these statements to arbitrary $n \geq 4$.

\subsubsection{General $n$}

The discussion for arbitrary $n$ follows closely the $n=4$ case. Indeed the main observation is that after solving the equivalence relations (3.6) and (3.7) the characteristics of the gauge invariance condition have the same structure as for $n=3,4$ :

$$
\frac{d y_{1}^{i}}{d \sigma}=w^{1 i}, \quad \frac{d z_{1}^{i}}{d \sigma}=y_{i}^{1},
$$

where $i \neq 1$. Notice that the above structure of the characteristics is not affected by the choice of independent variables $\Xi$, though upon a particular choice some of $y_{i}^{1}$ become linear combinations of the rest. Instead, $y_{i}^{1}$ can never be related to $y_{1}^{i}$ by the equivalence relations (3.6) and (3.7). Therefore, it is safe to work with the linearly dependant variables without specifying a basis.

The general solution of the gauge invariance conditions then reads for arbitrary $n$ as

$$
C^{(n)}=\mathcal{K}^{(n)}\left(h_{i j}, h_{i}^{j}, h^{i j}, c_{a}\right),
$$

where $c_{a}$ are all constant characteristics and where $h_{i j}, h_{i}^{j}$ and $h^{i j}$ are again defined as (3.24) but now $i, j$ runs from 2 to $n$. As in the $n=4$ case, not all $h$-structures are independent but they are subject to linear relations,

$$
\sum_{i=2}^{n} h^{k i}=0, \quad \sum_{i=2}^{n} h_{k}^{i}=0 .
$$

In the end, we conclude that the result for $n=4$ straightforwardly generalises to any $n$. At this point a few comments are in order:

- All $n$-pt couplings gauge invariant with respect to one massless higher-spin field, $\phi\left(x_{1}, u_{1}\right)$ here, are gauge invariant off-shell (again due to the fact that $y_{1}^{i}$ structure is not associated to a constant characteristic) for $n \geq 4$.

- Since such gauge invariant $n$-pt couplings are polynomials of $h$-structures (3.24) which are based on curls (3.26), the coupling function $\mathcal{K}^{(n)}$ involves $\partial_{u_{1}}$ only through the curl $\left[\partial_{x_{1}} \partial_{u_{1}}\right]^{\mu \nu}$. In other words, the components of the massless higher-spin field are all saturated by curls. From this, we can conclude that the massless higher-spin field enters to such couplings only through the form of linearised curvature. 
- So far we assumed that all the fields entering to the interaction are massless, and this assumption was used in (3.7). When some of the fields become massive, only the relation (3.7) gets modified to the massive mass-shell condition. However this relation is never used in the derivation of the solution, so our solution (3.32) is valid irrespectively of the mass of the other fields but the massless field $\phi\left(x_{1}, u_{1}\right)$. Remark that this was not the case for the $3 p t$ coupling of a massless field to two massive fields with equal mass where the $y_{1}^{i}$ characteristics collapses to a constant so can appear as an independent solution.

\subsection{Comments on dimensional-dependent identities}

So far in our analysis we have been working in arbitrary dimensions and have not considered dimensional dependent identities (DDIs) generated by over-antisymmetrisations of a number of indices greater than the space-time dimension (see e.g. [36, 47, 55-59] for related discussions). For the homogeneous $n$-pt coupling of totally symmetric fields, DDIs can be generated as

$$
\begin{aligned}
\mathrm{A}_{\left[k_{1} \cdots k_{q}\right]\left[l_{q+1} \cdots l_{d+1}\right]}^{\left[i_{1} \cdots i_{p}\right]\left[j_{p+1} \cdots j_{d+1}\right]}= & {\left[\left(\partial_{u_{i_{1}}}\right)_{\left[\mu_{1}\right.} \cdots\left(\partial_{u_{i_{p}}}\right)_{\mu_{p}}\left(\partial_{x_{j_{p+1}}}\right)_{\mu_{p+1}} \cdots\left(\partial_{x_{j_{d+1}}}\right)_{\left.\mu_{d+1}\right]}\right] \times } \\
& \times\left[\left(\partial_{u_{k_{1}}}\right)^{\left[\mu_{1}\right.} \cdots\left(\partial_{u_{k_{q}}}\right)^{\mu_{q}}\left(\partial_{x_{l_{q+1}}}\right)^{\mu_{q+1}} \cdots\left(\partial_{x_{l_{d+1}}}\right)^{\left.\mu_{d+1}\right]}\right] \equiv 0,
\end{aligned}
$$

where $i_{\bullet}, j_{\bullet}, k_{\bullet}, l_{\bullet}$ take values in $\{1, \ldots n\}$. Making use of the product identities of LeviCivita symbols, DDIs can be expressed as degree $d+1$ polynomials of $z_{i j}, y_{i}^{j}$ and $w^{i j}$, and these polynomials are identically zero. For the existence of DDIs, as is clear from the expression (3.34), the number of $\partial_{u_{i}}$ and $\partial_{x_{i}}$ should not be smaller than $d+1$. Taking into account the total derivative, this implies that non-trivial dimensional DDIs arise only for the $n$-point interactions with

$$
n \geq \frac{d+2}{2} .
$$

This bound is in agreement with the existence of DDIs for 3pt couplings in $d \leq 4$ and with the existence of DDIs for 4 pt couplings in $d \leq 6$. A similar counting gives a bound for couplings proportional to the $\epsilon$-tensor. They can exist only if $2 n-1 \geq d$ implying that the highest dimension in which a parity violating quartic interaction exist is $d=7$. Similarly the highest dimension for a parity violating cubic interaction is $d=5$. In any higher-dimension the epsilon tensor cannot be used to contract non-trivial quartic or cubic interactions respectively. ${ }^{11}$

It is clear from its definition (3.34) that the gauge variation of a DDI is a DDI, so the gauge variation of a coupling involving a DDI also involves a DDI: the gauge variation of an identically vanishing coupling vanishes identically. Below we will argue that if $n \geq 4$

\footnotetext{
${ }^{11}$ The fact that $d=7$ is the highest dimension in which $\epsilon$ tensor contributes to 4 pt structures may have non-trivial implications for the bootstrap program and is consistent with the fact that bootstrap equations for CFT correlators involving symmetric tensors become redundant in $d \leq 6$. This discontinuity in the number of independent bootstrap equations arising at the level of stress tensor correlators/totally symmetric fields might be one of the reason why it is so hard to construct non-trivial interacting unitary CFTs above $d=6$.
} 
also the opposite holds: if the gauge variation of a certain coupling is proportional to a DDI, then the coupling itself must be proportional to a DDI.

In order to understand the situation more clearly, let us begin by removing the ambiguities of field redefinitions and total derivaties in the TT part of the $n$-point coupling $C^{(n)}(w, y, z)$. Having in mind to analyse the gauge invariance for the first field, we can restrict $C^{(n)}$ to depend only on the following set of variables,

$$
\begin{aligned}
A= & \left\{w^{i j} \mid 1 \leq i<j \leq n-2\right\} \cup\left\{w^{i(n-1)} \mid 1 \leq i \leq n-3\right\} \\
& \cup\left\{y_{i}^{j} \mid 1 \leq i \neq j \leq n-1\right\} \cup\left\{y_{n}^{j} \mid 1 \leq j \leq n-2\right\} \\
& \cup\left\{z_{i j} \mid 1 \leq i<j \leq n\right\} .
\end{aligned}
$$

Then the gauge variation with respect to the first field is

$$
\left(\delta^{(0)} C^{(n)}\right)(w, y, z)=\left(\sum_{i=2}^{n} y_{i}^{1} \partial_{z_{1 i}}+\sum_{j=2}^{n-1} w^{1 j} \partial_{y_{1}^{j}}\right) C^{(n)}(w, y, z) .
$$

Note that the gauge variation introduces $y_{i}^{1}$ with $2 \leq i \leq n$ and $w^{1 j}$ with $2 \leq j \leq n-1$. The former is already in the set $A$ (3.36), whereas the latter is so only when $n \geq 4$. Hence, one can now distinguish two cases: (i) $w^{1 j} \neq 0$ which happens for $n \geq 4$, and (ii) $w^{1 j} \approx 0$ which happens for $n=3$.

Case $n \geq 4$. In this case, we just need to solve the equation,

$$
\left(\sum_{i=2}^{n} y_{i}^{1} \partial_{z_{1 i}}+\sum_{j=2}^{n-1} w^{1 j} \partial_{y_{1}^{j}}\right) C^{(n)}(w, y, z)=0 .
$$

The function $C^{(n)}(w, y, z)$ can be expressed as

$$
C(w, y, z)=C_{\mu_{1}^{1} \cdots \mu_{r_{1}}^{1} ; \nu_{1}^{1} \cdots \nu_{s_{1}}^{1} ; \cdots}\left(\partial_{x_{1}}^{\mu_{1}^{1}} \cdots \partial_{x_{1}}^{\mu_{r_{1}}^{1}}\right)\left(\partial_{u_{1}}^{\nu_{1}^{1}} \cdots \partial_{u_{1}}^{\nu_{s_{1}}^{1}}\right) \cdots,
$$

in terms of the tensor $C_{\mu_{1}^{1} \cdots \mu_{r_{1}}^{1} ; \nu_{1}^{1} \cdots \nu_{s_{1}}^{1} ; \ldots}$. The differential equation (3.38) is equivalent to the Young symmetrization condition of the tensor,

$$
C_{\left(\mu_{1}^{1} \cdots \mu_{r_{1}}^{1} ; \nu_{1}^{1}\right) \nu_{2}^{1} \cdots \nu_{s_{1}}^{1} ; \cdots}=0 .
$$

Let us look into this point more closely. The coupling tensor $C_{\mu_{1}^{1} \cdots \mu_{r_{1}}^{1} ; \nu_{1}^{1} \cdots \nu_{s_{1}}^{1} ; \cdots}$ is symmetic in each of $\mu_{1}^{i} \cdots \mu_{r_{i}}^{i}$ and $\nu_{1}^{j} \cdots \nu_{s_{j}}^{j}$, hence it has the symmetry of the Young tableaux,

$$
\begin{aligned}
& C_{\mu_{1}^{1} \cdots \mu_{r_{1}}^{1} ; \nu_{1}^{1} \cdots \nu_{s_{1}}^{1} ; \mu_{1}^{2} \cdots \mu_{r_{2}}^{2} ; \cdots ; \nu_{1}^{n} \cdots \nu_{s_{n}}^{n}} \\
& \sim \mu_{1}^{1} \cdots \mu_{r_{1}}^{1} \otimes \nu_{1}^{1} \cdots \\
& \hline
\end{aligned}
$$

The above tensor product can be decomposed into irreducible representations by successively applying the Littlewood-Richardson rule. In the end, we will obtain the direct sum,

$$
\bigoplus_{\mathbb{Y} \in Y} \mathbb{Y}
$$


where $Y$ is the set of the Young tableaux appearing in the decomposition of (3.41). Eventually, each $\mathbb{Y}$ corresponds to a possible interaction vertex, but we should impose a few more restrictions.

- The coupling tensor $C_{\mu_{1}^{1} \cdots \mu_{s_{1}}^{1} ; \nu_{1}^{1} \cdots \nu_{p_{1}}^{1} ; \ldots}$ is made by the metric tensors $\eta_{\nu^{i} \nu^{j}}, \eta_{\mu^{i} \nu^{j}}$ and $\eta_{\mu^{i} \mu^{j}}$ due to Lorentz invariance. This was already reflected in the choice of the variables $w, y, z$,

$$
w^{i j}=\eta_{\nu^{i} \nu^{j}} \partial_{x_{i}}^{\nu^{i}} \partial_{x_{j}}^{\nu^{j}}, \quad y_{i}^{j}=\eta_{\mu^{i} \nu^{j}} \partial_{u_{i}}^{\mu^{i}} \partial_{x_{j}}^{\nu^{j}}, \quad z_{i j}=\eta_{\mu^{i} \mu^{j}} \partial_{u_{i}}^{\mu^{i}} \partial_{u_{j}}^{\mu^{j}} .
$$

Therefore, not all Young tableaux $\mathbb{Y} \in Y$ suit the coupling tensor, but the ones that can be found in the tensor product decompositions of the metric tensors. It is known that the Young tableaux appearing in this decomposition have rows with an even number of boxes: let us call the set of such Young tableaux as $Y_{\text {even }}$. For any Young tableau $\mathbb{Y} \in Y_{\text {even }}$, there is a unique (up to an overall constant) tensor $\eta_{\mathbb{Y}}$ of the corresponding symmetry made by metric tensors, and the coupling tensor can be expressed as a linear combination of $\eta_{\mathbb{Y}}$ :

$$
C_{\mu_{1}^{1} \cdots \mu_{r_{1}}^{1} ; \nu_{1}^{1} \cdots \nu_{s_{1}}^{1} ; \cdots}=\sum_{\mathbb{Y} \in Y_{\text {even }}} \alpha_{\mathbb{Y}} \eta_{\mathbb{Y}} .
$$

Here $\alpha_{\mathbb{Y}}$ are coefficients parameterising the linear combination of couplings.

- The function $C(w, y, z)$ is restricted to the variables $A$ (3.36). This means that the tensor $C_{\mu_{1}^{1} \cdots \mu_{r_{1}}^{1} ; \nu_{1}^{1} \ldots}$ does not involve $\eta_{\bullet \bullet}$ corresponding to the omitted variables. Without loss of generality, this will reduce possible form of the coupling tensor to

$$
C_{\mu_{1}^{1} \cdots \mu_{r_{1}}^{1} ; \nu_{1}^{1} \cdots}=\sum_{m} c_{m} B_{\mu_{1}^{1} \cdots \mu_{r_{1}}^{1} ; \nu_{1}^{1} \cdots}
$$

where

$$
B_{\mu_{1}^{1} \cdots \mu_{r_{1}}^{1} ; \nu_{1}^{1} \ldots}^{m}=\sum_{\mathbb{Y} \in Y_{\text {even }}} \beta_{\mathbb{Y}}^{m} \eta_{\mathbb{Y}}
$$

are the most general linear combinations of $\eta_{\mathbb{Y}}$ which do not involve $\eta_{\bullet \bullet}$ corresponding to the omitted variables. Hence $B_{\mu_{1}^{1} \cdots \mu_{r_{1}}^{1} ; \nu_{1}^{1} \ldots}$ form the basis of the coupling tensor (but we did not impose yet the gauge invariance condition). A very simple way to understand the basis tensors $B_{\mu_{1}^{1} \cdots \mu_{r_{1}}^{1} ; \nu_{1}^{1} \ldots}^{m}$ is viewing them as the monomial basis of the variables in $A$ so that they span the space of function $C$.

- To have a concrete idea on the above points, let us consider a toy example of $C_{\mu_{1} \mu_{2} ; \nu_{1} \nu_{2}}$ :

$$
\begin{aligned}
& \begin{array}{l|l|l|l|l|}
C_{\mu_{1} \mu_{2} ; \nu_{1} \nu_{2}} \sim \mu_{1} & \mu_{2} \\
\hline
\end{array} \\
& =\begin{array}{|l|l|l|l|}
\hline \mu_{1} & \mu_{2} & \nu_{1} & \nu_{2} \\
\hline \nu_{2}
\end{array} \oplus \begin{array}{|l|l|l|}
\hline \mu_{1} & \mu_{2} & \nu_{1} \\
\hline \nu_{2}
\end{array} \oplus \begin{array}{|l|l|l|}
\hline \mu_{1} & \mu_{2} & \nu_{2} \\
\hline \nu_{1} & &
\end{array} \oplus \begin{array}{|l|l|}
\hline \mu_{1} & \mu_{2} \\
\hline \nu_{1} & \nu_{2} \\
\hline
\end{array} .
\end{aligned}
$$


Among the above, the Young tableaux in $Y_{\text {even }}$ are

$$
\begin{array}{|l|l|l|l|}
\hline \mu_{1} & \mu_{2} & \nu_{1} & \nu_{2} \\
\hline
\end{array} \oplus \begin{array}{|l|l|}
\hline \mu_{1} & \mu_{2} \\
\hline \nu_{1} & \nu_{2} \\
\hline
\end{array}
$$

and the corresponding $\eta_{\mathbb{Y}}$ tensors are

$$
\begin{aligned}
\eta_{\underline{\mu_{1}\left|\mu_{2} \nu_{1}\right| \nu_{2}}} & =\frac{1}{3}\left(\eta_{\mu_{1} \mu_{2}} \eta_{\nu_{1} \nu_{2}}+\eta_{\mu_{1} \nu_{2}} \eta_{\nu_{1} \mu_{2}}+\eta_{\mu_{1} \nu_{1}} \eta_{\mu_{2} \nu_{2}}\right), \\
\eta_{\substack{\mu_{1} \mid \mu_{2} \\
\nu_{1} \nu_{2}}} & =\frac{1}{3}\left(2 \eta_{\mu_{1} \mu_{2}} \eta_{\nu_{1} \nu_{2}}-\eta_{\mu_{1} \nu_{2}} \eta_{\nu_{1} \mu_{2}}-\eta_{\mu_{1} \nu_{1}} \eta_{\mu_{2} \nu_{2}}\right) .
\end{aligned}
$$

Therefore, we first find

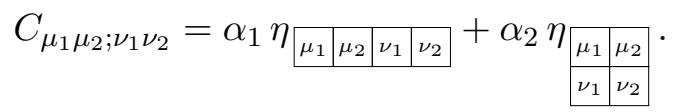

Impose the condition that the tensor $C_{\mu_{1} \mu_{2} ; \nu_{1} \nu_{2}}$ does not involve, say $\eta_{\mu_{1} \mu_{2}}$, the coupling tensor is reduced to

$$
\begin{aligned}
& C_{\mu_{1} \mu_{2} ; \nu_{1} \nu_{2}}=c_{1} B_{\mu_{1} \mu_{2} ; \nu_{1} \nu_{2}}^{1}, \\
& B_{\mu_{1} \mu_{2} ; \nu_{1} \nu_{2}}^{1}=2 \eta_{\underline{\mu_{1}\left|\mu_{2}\right| \nu_{1} \mid \nu_{2}}}-\eta_{\substack{\mu_{1}\left|\mu_{2} \\
\hline \nu_{1}\right| \nu_{2}}}=\eta_{\mu_{1} \nu_{2}} \eta_{\nu_{1} \mu_{2}}+\eta_{\mu_{1} \nu_{1}} \eta_{\mu_{2} \nu_{2}} .
\end{aligned}
$$

Now we impose the condition (3.40). We realise the symmetrization of indices $\mu_{1}^{1} \cdots \mu_{r_{1}}^{1} \nu_{1}^{1}$ as the action of the operator $\mathbf{S}$ on the tensor:

$$
C_{\left(\mu_{1}^{1} \cdots \mu_{r_{1}}^{1} ; \nu_{1}^{1}\right) \nu_{2}^{1} \ldots}=\mathrm{S} C_{\mu_{1}^{1} \cdots \mu_{r_{1}}^{1} ; \nu_{1}^{1} \ldots}=\sum_{m} c_{m} \mathrm{~S} B_{\mu_{1}^{1} \cdots \mu_{r_{1}}^{1} ; \nu_{1}^{1} \ldots}^{m}
$$

and

$$
\mathrm{S} B_{\mu_{1}^{1} \cdots \mu_{r_{1}}^{1} ; \nu_{1}^{1} \ldots}^{m}=\sum_{\mathbb{Y} \in Y_{\text {even }}} \beta_{\mathbb{Y}}^{m} \mathrm{~S} \eta_{\mathbb{Y}} .
$$

Young tableaux of the same Young diagram form an irreducible representation under the symmetric group, hence

$$
\mathrm{S} \eta_{\mathbb{Y}}=\sum_{\mathbb{Y}^{\prime} \in Y_{\mathrm{even}}} \sigma_{\mathbb{Y}, \mathbb{Y}^{\prime}} \eta_{\mathbb{Y}^{\prime}}
$$

where the coefficients $\sigma_{\mathbb{Y}, \mathbb{Y}^{\prime}}$ vanish unless the Young tableaux $\mathbb{Y}^{\prime}$ and $\mathbb{Y}$ have the same shape, i.e. Young diagram. The above equation determines how each basis tensor $B_{\mu_{1}^{1} \cdots \mu_{r_{1}}^{1} ; \nu_{1}^{1} \cdots}^{m}$ transforms under $\mathrm{S}$. The fact that the symmetrization $\mathrm{S}$ does not introduce any new variables means that $S$ is an endomorphism of the space of tensors spanned by $B_{\mu_{1}^{1} \cdots \mu_{r_{1}}^{1} ; \nu_{1}^{1} \ldots}^{m}:^{12}$

$$
\mathrm{S} B_{\mu_{1}^{1} \cdots \mu_{r_{1}}^{1} ; \nu_{1}^{1} \ldots}^{m}=\sum_{m^{\prime}} M_{m, m^{\prime}} B_{\mu_{1}^{1} \cdots \mu_{r_{1}}^{1} ; \nu_{1}^{1} \ldots}^{m^{\prime}}
$$

\footnotetext{
${ }^{12}$ If we regard the tensors $B_{\mu_{1}^{1} \cdots \mu_{r_{1}}^{1} ; \nu_{1}^{1} \ldots}^{m}$ as monomials of the variables in $A$, one should keep in mind that such a tensor corresponds to different monomials depending on whether it is viewed as a basis of coupling function or a basis of gauge variation of coupling function.
} 
In other words, the vector space $V$ of coupling tensors satisfy $\mathrm{S} V \subset V$. Therefore, the condition (3.40) is equivalent to finding the kernel of the matrix $S_{m, m^{\prime}}$ :

$$
\sum_{m} c_{m} S_{m, m^{\prime}}=0
$$

At this point, let us come back to our original question: whether there can be any coupling $C$ whose gauge variation is a DDI but itself is not. In the our setting, DDIs are simply,

$$
\eta_{\mathbb{Y}}=0, \quad \forall \mathbb{Y} \in Y_{\mathrm{DDI}}
$$

where $Y_{\text {DDI }} \subset Y_{\text {even }}$ is the set of Young tableaux having more than $d$ rows. Suppose we have a coupling tensor $C_{\mu_{1}^{1} \cdots \mu_{r_{1}}^{1} ; \nu_{1}^{1} \ldots}$ satisfying

$$
\mathrm{S} C_{\mu_{1}^{1} \cdots \mu_{r_{1}}^{1} ; \nu_{1}^{1} \ldots}=\sum_{\mathbb{Y} \in Y_{\mathrm{DDI}}} d_{\mathbb{Y}} \eta_{\mathbb{Y}}
$$

Since the symmetrization $S$ is a projection, $S^{2}=S$, we have

$$
\mathrm{S}\left(\sum_{\mathbb{Y} \in Y_{\mathrm{DDI}}} d_{\mathbb{Y}} \eta_{\mathbb{Y}}\right)=\sum_{\mathbb{Y} \in Y_{\mathrm{DDI}}} d_{\mathbb{Y}} \eta_{\mathbb{Y}} .
$$

This implies that to such a coupling tensor $C_{\mu_{1}^{1} \cdots \mu_{r_{1}}^{1} ; \nu_{1}^{1} \ldots}^{m}$ corresponds an ordinary one (that is, a coupling gauge invariant without relying on DDI),

$$
\tilde{C}_{\mu_{1}^{1} \cdots \mu_{r_{1}}^{1} ; \nu_{1}^{1} \ldots}=C_{\mu_{1}^{1} \cdots \mu_{r_{1}}^{1} ; \nu_{1}^{1} \ldots}-\sum_{\mathbb{Y} \in Y_{\mathrm{DDI}}} d_{\mathbb{Y}} \eta_{\mathbb{Y}}, \quad \mathrm{S} \tilde{C}_{\mu_{1}^{1} \cdots \mu_{r_{1}}^{1} ; \nu_{1}^{1} \cdots}=0
$$

What is important here is that $\sum_{\mathbb{Y} \in Y_{\mathrm{DDI}}}$ can be viewed as a (trivial) coupling tensor since it is a linear combination of $B_{\mu_{1}^{1} \cdots \mu_{r_{1}}^{1} ; \nu_{1}^{1} \ldots}^{m}$ (this is due to (3.57) and to the fact that $\sum_{\mathbb{Y} \in Y_{\mathrm{DDI}}} d_{\mathbb{Y}} \eta_{\mathbb{Y}}$ is in the image of $\mathrm{S}$ ).

This issue can be also understood from a slightly different point of view: recall that the action $S$ does not change the Young diagram, hence if we begin with the basis $B_{\mu_{1}^{1} \cdots \mu_{r_{1}}^{1} ; \nu_{1}^{1} \cdots}^{m}$ where we delete all $\eta_{\mathbb{Y} \in Y_{\mathrm{DDI}}}{ }^{13}$ then the symmetrization action $\mathrm{S}$ will never generate any new $\eta_{\mathbb{Y} \in Y_{\mathrm{DDI}}}$. In either way, we can conclude that it is not possible to have a coupling tensor $C_{\mu_{1}^{1} \cdots \mu_{r_{1}}^{1} ; \nu_{1}^{1} \ldots}$ which is not a (linear combination of) DDI, but $C_{\left(\mu_{1}^{1} \cdots \mu_{r_{1}}^{1} ; \nu_{1}^{1}\right) \ldots}$ is.

Let us close the analysis of the $n \geq 4$ case by showing how the kernel can be identified in a constructive manner. In fact, the condition (3.40) can be solved at the stage of applying Littlewood-Richardson rule to the first two Young tableaux: we restrict to

$$
\begin{aligned}
& C_{\mu_{1}^{1} \ldots \mu_{r_{1}}^{1} ; \nu_{1}^{1} \ldots \nu_{s_{1}}^{1} ; \mu_{1}^{2} \ldots \mu_{r_{2}}^{2} ; \cdots ; \nu_{1}^{n} \ldots \nu_{s_{n}}^{n}}
\end{aligned}
$$

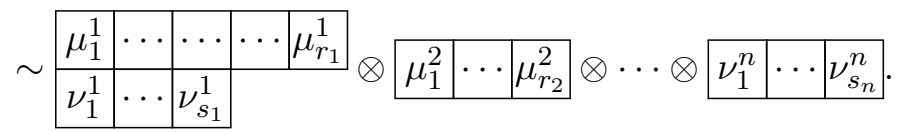

\footnotetext{
${ }^{13}$ This step can only introduce some linear dependencies in $B_{\mu_{1}^{1} \cdots \mu_{r_{1}}^{1} ; \nu_{1}^{1} \ldots}^{m}$ 's. Hence, we can just reduce the coefficient $c_{m}$ accordingly.
} 
By applying further the Littlewood-Richardson rules, the direct sum, $\bigoplus_{\mathbb{Y} \in \tilde{Y}} \mathbb{Y}$ where $\tilde{Y} \subset$ $Y$ is the set of Young tableaux satisfying $\mathbf{S} \mathbb{Y}=0$. Then, we further restrict to the set $\tilde{Y}_{\text {even }}$ even-row Young tableaux and eventually take the subspace which do not involve $\eta_{\bullet \bullet}$ corresponding to the omitted variables.

Case $\boldsymbol{n}=3$. In this case, the arguments of $n \geq 4$ do not apply any more, and there may exist non-trivial couplings whose gauge variation vanishes up to DDIs. From the condition (3.35), the relevant dimensions are $d \leq 4$. In $d=4$, some massless cubic interactions become proportional to DDI leaving always two independent interactions for each $s_{1}, s_{2}, s_{3}$ couplings, but there is no interaction whose gauge variation is a DDI. In $d=3$, the Chern-Simons formulation tells us that we have two derivative $s-s-2$ interactions, whose analogue cannot be found in dimensions higher than 3. In fact, these two-derivative interactions are precisely the one whose gauge variation is proportional to DDI. Systematic analysis of $3 \mathrm{~d}$ interaction vertices has been carried out in $[56,57]$ (see also $[36,55,58,59]$ ) and all interactions whose gauge variation becomes a DDI have been explicitly classified. Here, we revisit this story to see more concretely why this type of interactions exist only for the cubic interactions but not for higher order interactions.

We begin again with the set of variable $A(3.36)$, which reduces to

$$
A=\left\{y_{1}^{2}, y_{2}^{1}, y_{3}^{1}, z_{12}, z_{13}, z_{23}\right\}
$$

The gauge variation of the coupling function $C$ is

$$
\left(\delta^{(0)} C^{(3)}\right)(w, y, z)=\left(y_{2}^{1} \partial_{z_{12}}+y_{3}^{1} \partial_{z_{13}}+w^{12} \partial_{y_{1}^{2}}\right) C(y, z),
$$

and we find that the gauge variation introduces back the variable $w^{12}$ which has been discarded from the set $A$. Hence, this means that the vector space $V$ of coupling tensors is not invariant under the action of $\mathrm{S}: \mathrm{S} V \not \subset V$. For the subsequent analysis, it is useful explicitly consider couplings involving $w^{12}$. But the monomial functions involving $w^{12}$ correspond to different coupling tensors depending on whether one considers a coupling or a gauge variation of a coupling. This is because additional $w^{12}$ monomials are generated by the gauge variation. Hence we have schematically

$$
V \oplus W=\bar{V} \oplus \bar{W} .
$$

where $V$ and $\bar{V}$ are the vector spaces of tensors associated with couplings and variation of couplings which do not involve $\eta_{\bullet \bullet}$ corresponding to $w^{12}$. And $W$ and $\bar{W}$ are the analoguous vector spaces where the tensors do involve $\eta_{\bullet \bullet}$ corresponding to $w^{12}$. In terms of these, we have

$$
\mathrm{S} V \subset \bar{V} \oplus \bar{W}
$$

If the basis tensors of the space $V, \bar{V}, W$ and $\bar{W}$ are denoted by $B_{\mu_{1}^{1} \cdots \mu_{r_{1}}^{1} ; \nu_{1}^{1} \ldots}^{m}, D_{\mu_{1}^{1} \ldots \mu_{r_{1}}^{1} ; \nu_{1}^{1} \ldots}^{a}$, $\bar{B}_{\mu_{1}^{1} \cdots \mu_{r_{1}}^{1} ; \nu_{1}^{1} \ldots}^{\bar{m}}$ and $\bar{D}_{\mu_{1}^{1} \cdots \mu_{r_{1}}^{1} ; \nu_{1}^{1} \ldots}^{\bar{a}}$, respectively, then (3.67) implies

$$
\mathrm{S} B_{\mu_{1}^{1} \cdots \mu_{r_{1}}^{1} ; \nu_{1}^{1} \ldots}^{m}=\sum_{\bar{m}} S_{m, \bar{m}} \bar{B}_{\mu_{1}^{1} \cdots \mu_{r_{1}}^{1} ; \nu_{1}^{1} \ldots}^{\bar{m}}+\sum_{\bar{a}} S_{m, \bar{a}} \bar{D}_{\mu_{1}^{1} \cdots \mu_{r_{1}}^{1} ; \nu_{1}^{1} \ldots}^{\bar{a}}
$$


Let us consider now a coupling gauge invariant up to DDIs: the corresponding coupling tensor $C_{\mu_{1}^{1} \cdots \mu_{1} ; \nu_{1}^{1} \ldots}$ should satisfy

$$
\mathrm{S} C_{\mu_{1}^{1} \cdots \mu_{r_{1}}^{1} ; \nu_{1}^{1} \ldots}=\sum_{\mathbb{Y} \in Y_{\mathrm{DDI}}} d_{\mathbb{Y}} \eta_{\mathbb{Y}}+\sum_{i} d_{\bar{a}} \bar{D}_{\mu_{1}^{1} \cdots \mu_{r_{1}}^{1} ; \nu_{1}^{1} \ldots}^{\bar{a}} .
$$

Compared to the $n \geq 4$ analog (3.60), we appended the terms involving $\bar{D}_{\mu_{1}^{1} \cdots \mu_{r_{1}}^{1} ; \nu_{1}^{1} \ldots}^{\bar{a}}$ to incorporate the on-shell condition $w^{12} \approx 0$. We examine whether the term $\sum_{\mathbb{Y} \in Y_{\mathrm{DDI}}} d_{\mathbb{Y}} \eta_{\mathbb{Y}}$ in the left hand side of the equation can be removed by appropriately subtracting a coupling tensor $\Delta C_{\mu_{1}^{1} \cdots \mu_{r_{1}}^{1} ; \nu_{1}^{1} \ldots}$ which is a DDI by itself:

$$
\Delta C_{\mu_{1}^{1} \cdots \mu_{r_{1}}^{1} ; \nu_{1}^{1} \ldots}=\sum_{\mathbb{Y} \in Y_{\mathrm{DDI}}} \tilde{d}_{\mathbb{Y}} \eta_{\mathbb{Y}}, \quad \mathrm{S} \Delta C_{\mu_{1}^{1} \cdots \mu_{r_{1}}^{1} ; \nu_{1}^{1} \cdots}=\sum_{\mathbb{Y} \in Y_{\mathrm{DDI}}} d_{\mathbb{Y}} \eta_{\mathbb{Y}}
$$

Differently from the $n \geq 4$ case, the property (3.61) does not hold in this case, hence there is no universal way to get $\Delta C_{\mu_{1}^{1} \cdots \mu_{r_{1}}^{1} ; \nu_{1}^{1} \ldots}$ for each $C_{\mu_{1}^{1} \cdots \mu_{r_{1}}^{1} ; \nu_{1}^{1} \ldots}$ satisfying (3.69), and there are cases where $\Delta C_{\mu_{1}^{1} \cdots \mu_{r_{1}}^{1} ; \nu_{1}^{1} \ldots}$ does not exist and these cases correspond precisely to the couplings whose gauge invariance rely on DDI. ${ }^{14}$

To summarise, we have argued that for $n>3$ DDIs do not introduce new non-trivial solutions on top of the homogeneous equation solutions (3.32). The effect of DDIs for $n>3$ is therefore relegated to produce linear relations between a priori different homogeneous solutions. For example in $d=3$ all Weyl tensor vanish for $l \geq 2$ and the corresponding n-pt structure proportional to higher-spin fields therefore also vanishes. We conclude that in $d=3$ there cannot exist a non-vanishing parity preserving homogeneous solution with $n>3$ encoding a coupling of a massless higher-spin field to any massive/massless field of any spin, including scalars and spin-1.

\subsection{Combining solutions: more than one massless field}

So far we have focused for simplicity on the solution space for couplings which is gauge invariant with respect to a single external leg. When more than one field is massless, one should then consider the intersection of the corresponding solution spaces associated with each legs (see e.g. [52] for a detailed discussion of this at the $3 p t$ level). This is straightforward to analyse because we have shown that the gauge invariance of a coupling with respect to one field, say $\phi\left(x_{i}, u_{i}\right)$, allows the $\partial_{u_{i}}$ dependence only through the form of the curl $\left(\partial_{u_{i}}\right)^{[\mu}\left(\partial_{x_{i}}\right)^{\nu]}$. For instance, when all the fields are massless, all $\partial_{u_{i}}$ with $i=1, \ldots, n$ should enter through the curls. Then, the only allowed structures are the contractions between two curls:

$$
H_{i j}=2\left(\partial_{u_{i}}\right)_{[\mu}\left(\partial_{x_{i}}\right)_{\nu]}\left(\partial_{u_{j}}\right)^{[\mu}\left(\partial_{x_{j}}\right)^{\nu]}=z_{i j} w^{i j}-y_{j}^{i} y_{i}^{j},
$$

the contractions between one curl and $\left(\partial_{x_{j}}\right)_{\mu}\left(\partial_{x_{k}}\right)_{\nu}$ :

$$
H_{i}^{j k}=2\left(\partial_{u_{i}}\right)_{[\mu}\left(\partial_{x_{i}}\right)_{\nu]}\left(x_{j}\right)^{\mu}\left(x_{k}\right)^{\nu}=y_{i}^{j} w^{i k}-y_{i}^{k} w^{i j},
$$

\footnotetext{
${ }^{14}$ One may wonder the property $\mathrm{S}\left(\sum d_{\mathbb{Y}} \eta_{\mathbb{Y}}+\sum d_{\bar{a}} \bar{D}^{\bar{a}}\right)=\sum d_{\mathbb{Y}} \eta_{\mathbb{Y}}+\sum d_{\bar{a}} \bar{D}^{\bar{a}}$ might be useful in this argument. But, it just tells that $\sum d_{\bar{a}} \bar{D}^{\bar{a}}$ can be viewed as the coupling gauge invariant up to DDI. Remember that $\bar{D}^{\bar{a}}$ is a linear combination of $B^{m}$ and $D^{a}$, so $\sum d_{\bar{a}} \bar{D}^{\bar{a}}$ is a non-trivial on-shell coupling.
} 
and the contractions of $\partial_{x_{i}}$ 's, that is $w^{i j}$ 's. In the end, the solution space for the gauge invariant coupling of $n$ massless fields is

$$
C^{(n)}=K^{(n)}\left(H_{i j}, H_{i}^{j k}, w^{i j}\right) .
$$

Again, $H_{i}^{j k}$ and $w^{i j}$ are not all independent but satisfy

$$
\sum_{j=1}^{n} H_{i}^{j k} \sim 0, \quad \sum_{j=1}^{n} w^{i j} \sim 0 .
$$

An interesting observation that follows from the analysis of gauge invariant solutions we have done here is that all solutions found are in one-to-one correspondence with tensorial structures which can all be obtained from powers of spin- 1 and scalars couplings. ${ }^{15}$ This simply follows in this context from the fact that each of $H_{i j}$ and $H_{i}^{j k}$ are in one-to-one correspondence with gauge boson coupling to scalar field. Therefore this gives a different argument for the result obtained by a brute force analysis in [1].

In the following we will consider the generalisation of the flat-space analysis to (A)dS.

\section{Homogeneous solution in AdS}

The analysis of the homogeneous couplings in flat space can be straightforwardly generalised to the (A)dS case using the ambient space formalism. See [2] for some initial discussions on (A)dS homogeneous solutions using the ambient space formalism. $(A) d S_{D}$ space is the hypersurface $X^{2}=-L^{2}$ embedded in the ambient space $\mathbb{R}^{D+1}$, and the (A)dS fields are homogeneous, $\left(X \cdot \partial_{X}-U \cdot \partial_{U}+2-\mu\right) \Phi(X, U)=0$, and tangent, $\left(X \cdot \partial_{U} \Phi(X, U)=0\right.$. The starting point is again to express the homogeneous coupling $S_{h}^{(n)}$ through a function $C_{h}$ as

$$
\left.S_{h}^{(n)} \stackrel{\text { TT }}{=} \int_{(\mathrm{A}) \mathrm{dS}} C_{h}^{(n)}(W, Y, Z) \Phi_{1}\left(X_{1}, U_{1}\right) \cdots \Phi_{n}\left(X_{n}, U_{n}\right)\right|_{\substack{X_{i}=X \\ U_{i}=0}},
$$

where the variables $W^{i j}, Y_{i}^{j}$ and $Z_{i j}$ are the ambient space analogues of (3.3):

$$
W^{i j}=\partial_{X_{i}} \cdot \partial_{X_{j}}, \quad Y_{i}^{j}=\partial_{U_{i}} \cdot \partial_{X_{j}}, \quad Z_{i j}=\partial_{U_{i}} \cdot \partial_{U_{j}} \cdot
$$

Similarly to the flat space cases, $Y_{i}^{i}$ and $Z_{i i}$ are excluded by the transversality and tracelessness while $W^{i i}$ is removed by a field redefinition. However, the flat-space relations (3.6), given by the ambiguities of integration-by-parts, are now modified into the following constraints imposed on $C_{h}^{(n)}$ :

$$
\mathcal{D}_{i}^{Y} C_{h}^{(n)}(W, Y, Z)=0, \quad \mathcal{D}_{i}^{W} C_{h}^{(n)}(W, Y, Z)=0,
$$

\footnotetext{
${ }^{15}$ This observation was made at cubic order in [18] and extended to higher point amplitudes in [1] where it was also shown to be valid in the case of fermionic couplings. It can be considered as a generalisation of the double-copy structure of spinning homogeneous solutions.
} 
where $\mathcal{D}_{i}^{Y}$ and $\mathcal{D}_{i}^{W}$ are the differential operators,

$$
\begin{aligned}
\mathcal{D}_{i}^{Y} & =\sum_{k=1}^{n}\left[Y_{i}^{k}+\lambda\left(Z_{i k} \partial_{Y_{k}^{i}}+Y_{i}^{k} \partial_{W^{i k}}\right)\right] \\
\mathcal{D}_{i}^{W} & =\sum_{k=1}^{n}\left[W^{i k}+\lambda\left(2-Z_{i k} \partial_{Z_{i k}}+Y_{i}^{k} \partial_{Y_{i}^{k}}-Y_{k}^{i} \partial_{Y_{k}^{i}}+W^{i k} \partial_{W^{i k}}\right)\right] .
\end{aligned}
$$

Here, $\lambda$ is defined as ${ }^{16}$

$$
\int_{(\mathrm{A}) \mathrm{dS}} \lambda^{n}=\int d^{D+1} X \delta^{[n]}\left(X^{2}-L^{2}\right) .
$$

The operators $\mathcal{D}_{i}^{Y}$ and $\mathcal{D}_{j}^{W}$ commute among themselves and with each others. This property is manifest if one considers their origin,

$$
\mathcal{D}_{i}^{Y} \sim \partial_{U_{i}} \cdot\left(\sum_{k=1} \partial_{X_{k}}\right), \quad \mathcal{D}_{i}^{W} \sim \partial_{X_{i}} \cdot\left(\sum_{k=1} \partial_{X_{k}}\right) .
$$

Therefore, the numbers of independent variables $W, Y, Z$, is obviously the same as in flat space:

$$
(\# \text { of } W)=\frac{1}{2} n(n-3), \quad(\# \text { of } Y)=n(n-2), \quad(\# \text { of } Z)=\frac{1}{2} n(n-1) .
$$

The solutions $C_{h}^{(n)}$ of the above equations are in one-to-one correspondence to the TT part of all possible $n$-th order vertices.

The next step is to deal with the condition of gauge invariance $\delta^{(0)} S_{h}^{(n)} \approx 0$ in (A)dS. The lowest order gauge symmetry of (A)dS fields can be written in the ambient space formulation as $\delta \Phi(X, U)=U \cdot \partial_{X} E(X, U)$. Therefore, the gauge invariance of (4.1) up to an on-shell and TT conditions gives

$$
\left[C^{(n)}(W, Y, Z), U_{i} \cdot \partial_{X_{i}}\right] \approx \mathcal{D}_{i}^{\text {gauge }} C_{h}^{(n)}(W, Y, Z)=0 .
$$

where

$$
\mathcal{D}_{i}^{\text {gauge }}=\sum_{k=1}^{n}\left(Y_{k}^{i} \partial_{Z_{i k}}+W^{i k} \partial_{Y_{i}^{k}}\right) .
$$

Notice here that we have treated the $Y$ and $W$ variables as if they are all independent. This is consistent because the differential operator $\mathcal{D}_{i}^{\text {gauge }}$ in eq. (4.9) leaves the constrained surface given by eq. (4.5) invariant. ${ }^{17}$ This can be seen from the relations,

$$
\left[\mathcal{D}_{i}^{\text {gauge }}, \mathcal{D}_{j}^{Y}\right]=\delta_{i j} \mathcal{D}_{i}^{W}, \quad\left[\mathcal{D}_{i}^{\text {gauge }}, \mathcal{D}_{j}^{W}\right]=0,
$$

which are again obvious consequences of (4.7). Therefore, it is consistent to work with linearly dependent set of variables like we did in the flat-space analysis. The simple structure

\footnotetext{
${ }^{16}$ See e.g. $\S(5)$ of $[20]$ and $\S(3.4)$ of [2] with the replacement $\lambda \rightarrow \hat{\delta}$, for more details.

${ }^{17}$ This is another way of saying that as in flat space case the characteristics of the above equations are at most linear regardless the choice of representative for the equivalence relation (4.3).
} 
of the PDE (4.9) which is basically the same as the flat space equation makes it possible to identify its general solution precisely as in the flat space case: ${ }^{18}$

$$
C^{(n)}=K^{(n)}\left(H_{i j}, H_{i}^{j k}, W^{i j}\right)
$$

where $H_{i j}$ and $H_{i}^{j k}$ are defined by ${ }^{19}$

$$
H_{i j}=Z_{i j} W^{i j}-Y_{j}^{i} Y_{i}^{j}, \quad H_{i}^{j k}=Y_{i}^{j} W^{i k}-Y_{i}^{k} W^{i j} .
$$

It is interesting to observe how one might have obtained the above solution by a straightforward covariantisation of the flat-space solutions (3.71) and (3.72). It is indeed very easy to argue in general (see e.g. $[15,19])$ that for each (A)dS solution to the gauge invariance condition there exist a corresponding flat space solution in the limit $\Lambda \rightarrow 0$. The number of flat-space solution is therefore an upper bound to the number of (A)dS solutions. Since all flat-space $h$-solutions (3.32) can be trivially covariantised to the (A)dS ones, we can conclude that the (A)dS $h$-solutions also form a complete set for $n \geq 4$.

Only the $n=3$ case is different. Since some of $y_{i}^{j}$ variables are gauge invariant on-shell, there exist more solutions besides the $h$-structures. To find out all solutions, we first solve the linear dependency (4.5) by discarding half of the $Y_{i}^{j}$ 's and all the $W^{i j}$ 's. This will deform the equation (4.9) into

$$
\mathcal{D}_{1}^{\text {gauge }}=Y_{3}^{1} \partial_{Z_{31}}-Y_{2}^{3} \partial_{Z_{12}}+\lambda\left(Y_{3}^{1} \partial_{Y_{3}^{1}}-Y_{2}^{3} \partial_{Y_{2}^{3}}\right) \partial_{Y_{1}^{2}}
$$

whose solutions were analyzed in $[2,19,20,51]$ and provide all gauge invariant 3-pt couplings. ${ }^{20}$ Since the above solutions cover the entire solution space, the $h$-solutions should belong to it: some solutions of the above equation can be re-expressed as $h$-solutions (see e.g. [52] for additional details).

\section{Particular solutions, current exchanges \& locality}

Before concluding this note, we would like to spend a few lines by reviewing what is known about the particular solution to the Noether procedure $[1,21]$.

From the standard Noether procedure point of view, one is usually asked to directly find, under the locality assumption, a local particular solution. However this strategy proves to hide some conceptual simplicity. In the following, along the lines of [1], we shall not ask any further requirement on the particular solution with the aim of simplifying its

\footnotetext{
${ }^{18}$ Indeed the characteristics of the above equation are the same as in flat-space for $n>3$ since also in (A)dS it is always possible to fix a representative of the equivalence relation such that all characteristics are either linear or constant.

${ }^{19}$ We are focusing here for brevity directly on couplings of massless higher-spin field for which we must impose gauge invariance with respect to all external legs. In the case in which some of the field are massive one should also consider the AdS covariantisation of the additional $h$-structures we found in the flat space classification. This is straightforward in the ambient space.

${ }^{20}$ The radial reduction of these vertices has been also studied in [2, 49, 60-62].
} 
structure relating it to the lower point couplings. The questions about locality will be raised at a different but equivalent level in terms of the full coupling defined above as ${ }^{21}$

$$
S^{(n)}=S_{h}^{(n)}+S_{p}^{(n)},
$$

even though in the present letter we do not aim for this issue (we refer to [1, 2, 21]). Since the particular solution is always defined up to a homogeneous one, one can choose any solution of

$$
\delta^{(0)} S_{p}^{(n)} \approx-\left(\delta^{(1)} S^{(n-1)}+\cdots+\delta^{(n-3)} S^{(3)}\right) .
$$

Actually here exists a simple solution of the above equation - the $n$-point amplitude composed by all the $m$-point vertices with $m \leq n-1$.

To explain the point, let us use the diagramms where the quadratic and cubic actions are depicted as

$$
S^{(2)}\left(\Phi_{i}\right)=i \longmapsto i, \quad S^{(3)}\left(\Phi_{i}, \Phi_{j}, \Phi_{k}\right)=\bigodot_{k}^{i} j,
$$

and the lowest order gauge transformation as

$$
\delta_{i}^{(0)} \Phi_{i}=\quad i>\cdots \cdots \text { ○ } \longrightarrow i
$$

Here, each graph stands for a map from the fields depicted by inward edges to the field depicted by outward edges. The graphs corresponding to $S^{(n)}$ have only $n$ inward edges, hence stands for a functional of $n$ fields depicted by such edges. The graphs corresponding to $\delta_{i}^{(n)} \Phi_{j}$ has $n$ inward edges together with one outward edge and one blue dotted inward edge. The latter two indicate the output gauge variation and the gauge parameter, respectively. For instance, in the graph (5.4), the blue dotted inward edge and the black solid outward edge indicate, respectively, the gauge parameter $E_{i}$ and the gauge variation $\delta_{i}^{(0)} \Phi_{i}$, while the yellow vertex with two legs stands for the gradient operator, $U_{i} \cdot \partial_{X_{i}}$, implying that the output, $\delta_{i}^{(0)} \Phi_{i}$, is the gradient of the input, $E_{i}$, i.e. $\delta_{i}^{(0)} \Phi_{i}=U_{i} \cdot \partial_{X_{i}} E_{i}$. The relation $\delta^{(0)} S^{(3)}+\delta^{(1)} S^{(2)}=0$, or more precisely,

$$
\delta_{1}^{(0)} \Phi_{1} \frac{\delta S^{(3)}\left(\Phi_{1}, \Phi_{2}, \Phi_{3}\right)}{\delta \Phi_{1}}+\left[\delta_{1}^{(1)} \Phi_{2}\right]_{3} \frac{\delta S^{(2)}\left(\Phi_{2}\right)}{\delta \Phi_{2}}+\left[\delta_{1}^{(1)} \Phi_{3}\right]_{2} \frac{\delta S^{(2)}\left(\Phi_{3}\right)}{\delta \Phi_{3}}=0,
$$

can be expressed diagrammatically as

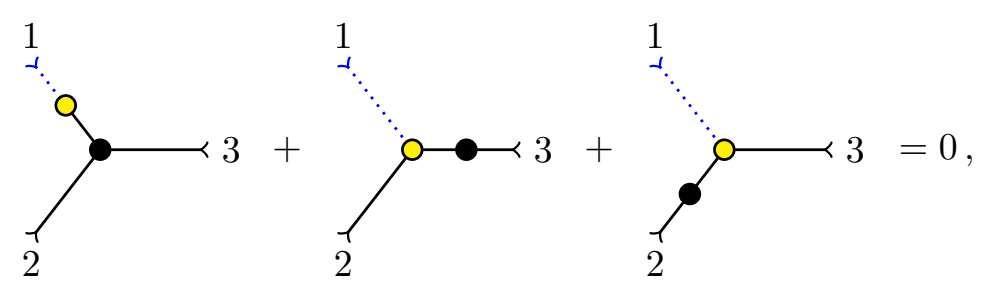

\footnotetext{
${ }^{21}$ In [21] locality was replaced by the condition that the homogeneous solutions form representations of the HS algebra. This allows to uniquely fix the homogeneous solutions on-shell. Locality remains an important condition since the off-shell field frame cannot be fixed in this way and leads to divergences.
} 
with $\left[\delta_{i}^{(1)} \Phi_{j}\right]_{k}$ — the part of the variation of the field $\Phi_{j}$ under the gauge symmetry of $i$-th field which is linear in $\Phi_{k}$ - depicted as

$$
\left[\delta_{i}^{(1)} \Phi_{j}\right]_{k}=\overbrace{k}^{\stackrel{i}{\lrcorner}} \longrightarrow j .
$$

Here, we used the rule,

$$
\longrightarrow i \longleftarrow=i \longmapsto
$$

Now we are ready to proceed to the 4-pt coupling whose particular solution can be chosen as the current-exchange part of the quartic amplitude:

$$
S_{p}^{(4)}\left(\Phi_{1}, \Phi_{2}, \Phi_{3}, \Phi_{4}\right)=
$$

where the white circle in the internal line is the $1 / \square$ propagator for the field $k$, hence satisfying

$$
\longrightarrow \text { - } \longrightarrow \longrightarrow+-0 \cdots 0 \cdots 0-\text {. }
$$

The second term of the right hand side of the equality indicates the divergence term. ${ }^{22}$ The gauge variation of $S_{p}^{(4)}(5.9)$ gives

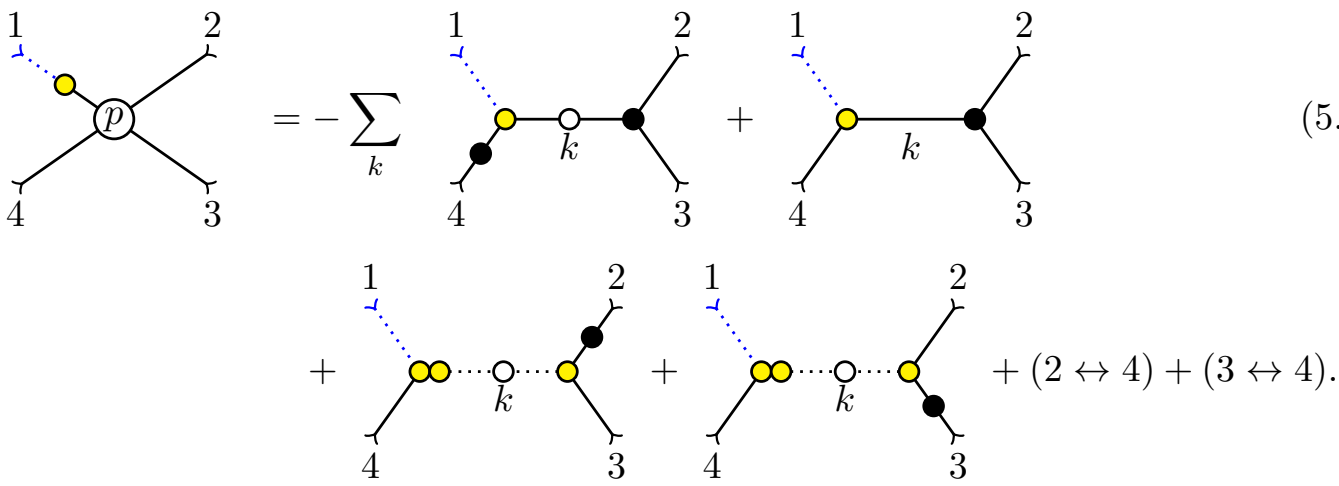

\footnotetext{
${ }^{22}$ Note that we can write the divergence term in terms of the notation (5.4). In particular the adjoint of (5.4) is obtained reflecting the diagram with respect to the vertical axis and is interpreted as a divergence.
} 
where we used (5.10) to get the diagrammes with two bullets. These diagrammes correspond to $\delta^{(1)} S^{(3)}$ while the diagrammes with more bullets to $\delta^{(2)} S^{(2)}$ with

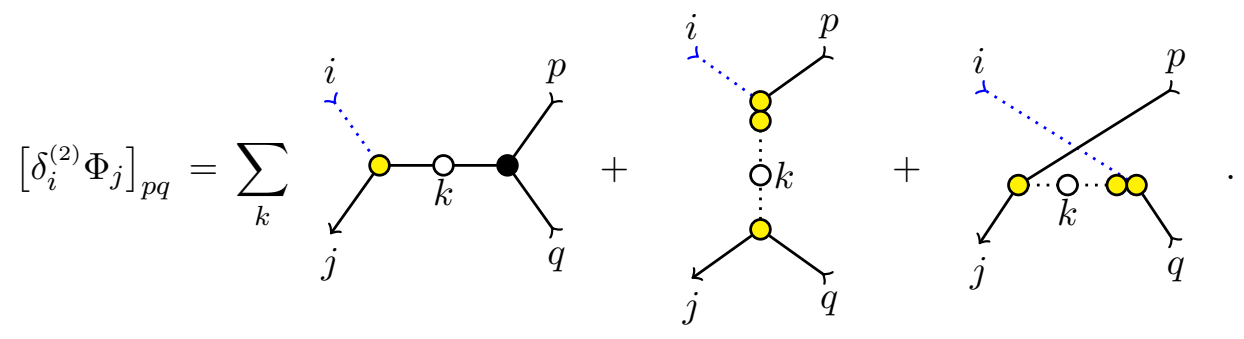

Notice that the above $\delta^{(2)}$ is generically non-local. Since we have shown that the homogeneous solution $S_{h}^{(4)}$ does not induce any $\delta^{(2)}$, this seems in contradiction with existence of any local theory. The point is that the latter statement holds only up to a field redefinition, which may be non-local as well but well-defined on shell. To summarise, a homogeneous solution $S_{h}^{(4)}$ resulting from a non-local field redefinition,

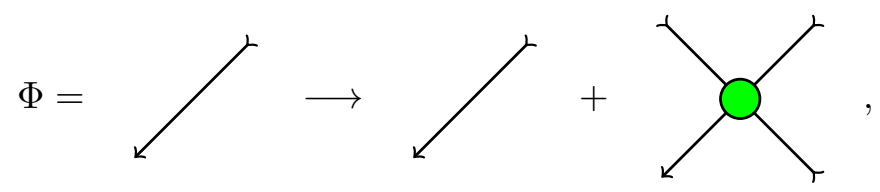

may compensate the non-local deformation of gauge transformation:

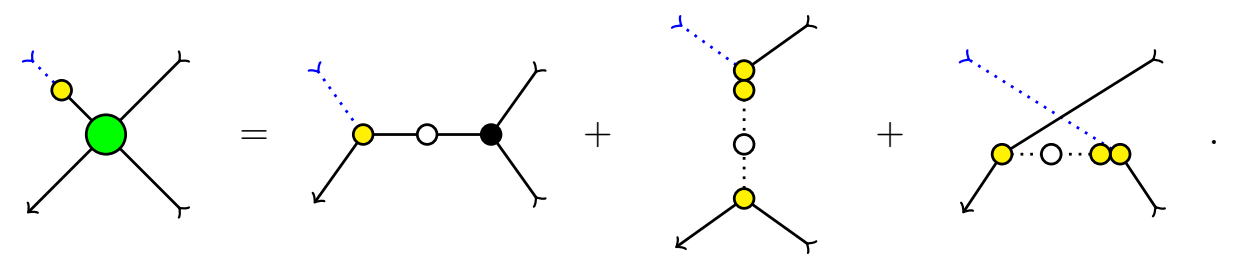

This is indeed what happens in all local theories. Appendix A contains an explicit treatment of this point through the example of Yang-Mills theory and Einstein Gravity. In the end, combining the particular solution $S_{p}^{(4)}(5.9)$ with a homogeneous solution $S_{h}^{(4)}$ - consisting of a $h$-coupling (3.73) and a non-local field redefinition - we obtain the most general form of consistent quartic couplings. In local theories, the non-localities in $S_{h}^{(4)}$ should cancel on-shell those in $S_{p}^{(4)}$, and this can happen only up to a suitable non-local but non-singular field redefinition. ${ }^{23}$

For higher-order interactions, the particular solutions $S_{p}^{(n)}$ can be found in the same manner as the quartic one, in terms of the homogeneous solutions $S_{h}^{(m)}$ with $m \leq n-1$.

\footnotetext{
${ }^{23}$ We stress here that such redefinitions are in one to one correspondence with non-local functionals which vanish on-shell. This is different from non-local redefinitions which are expressed in terms of singular functionals and can remove non-trivial couplings. Most importantly such singular redefinition should not be allowed and pose a question for the off-shell definition of any $n$-point coupling in a theory with infinitely many fields (see e.g. the discussion in [7, 21]).
} 
For instance, the quintic and the sextic couplings are given schematically by

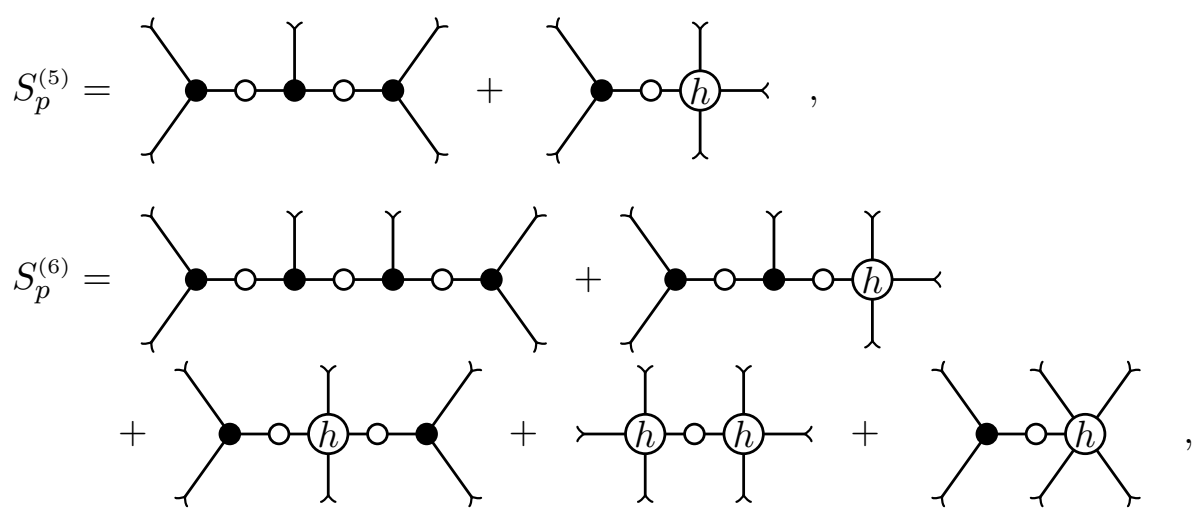

where the $h$-bullet vertices label the corresponding homogeneous solutions:

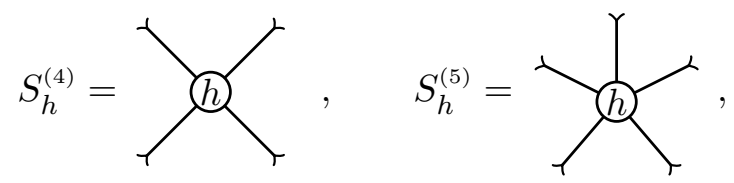

and the permutations of the external legs are suppressed for simplicity. From these particular solutions, one can extract the corresponding deformation of gauge transformations, $\delta^{(n-2)}$, which are generically non-local. If the theory under consideration is local, then this is equivalent to the existence of a non-singular, non-local redefinition which would remove all the non-local terms in $\delta^{(n-2)}$. If this field redefinition removes all terms without leaving any local remainders, the gauge algebra is at most linear in the fields, which is the case in YM theory and Gravity. ${ }^{24}$

Before adding more comments on the locality issue, let us briefly remark that the approach taken in this section did not assume the TT condition: the cubic coupling $S^{(3)}$ and the homogeneous solution $S^{(n)}$ may contain the divergence and trace terms. Even when we impose the on-shell conditions on the external legs, some of such non-TT terms survive and contribute to the construction of $S_{p}^{(m)}$. Such contribution is generically a contact contribution.

Let us make a few comments on the non-locality issues in higher-spin(-like) theories. As we explained above, if the sought theory is local, the homogeneous solution $S_{h}^{(n)}$ consisting of a $h$-coupling and a non-local field redefinition - can be uniquely determined because we can use all our freedom of non-local field redefinitions in removing the nonlocalities and this is possible only upon a unique choice of $h$-coupling. On the contrary, in non-local theories, it is, by definition, impossible to remove all the non-localities. Therefore, we can neither fix the $h$-coupling nor the non-local field-redefinition: we need a new criterion in order to avoid this ambiguity. For instance, one can forbid the coupling to involve any singularities of physical exchange. This condition allows for, besides local interactions, a vast range of non-local interactions. When there is a solution to this condition, it would

\footnotetext{
${ }^{24}$ Note that the deformations of the gauge transformations are linear only in the YM case while in the Gravity case they receive all orders deformations which resum to give the inverse metric. The gauge algebra is however fixed at the cubic level and does not receive further non-trivial corrections.
} 
fix, for instance, the $h$-coupling part (or the on-shell non-trivial part) of $S_{h}^{(4)}$ in terms of the cubic coupling $S^{(3)}$ (as shown in $[1,7]$ ). ${ }^{25}$ The last but not the least important point relevant to the discussions of this paragraph is the convergence issue in the sum $\sum_{k}$ in $S_{p}^{(4)}$, which may obscure even further the nature of the non-locality is $S_{p}^{(4)}$ (see e.g. $\S(4.3)$ of [7]).

We conclude this discussion stressing that a possibly interesting application of the results presented in this note is in the connection between the homogeneous solution to the Noether procedure in (A)dS and the $n$-point conformal structures of conserved currents. In this context gauge invariance in the bulk translates into current conservation on the boundary and this ambient space formulation could potentially be used in applications to CFT correlators with conserved spinning currents. We leave to the future a more detailed analysis of this connection and the possible applications to spinning correlators.

\section{Acknowledgments}

M.T. is grateful to Carlo Iazeolla and Per Sundell for useful discussions and especially to Charlotte Sleight for useful discussions and comments on the draft. This work was initiated at Scuola Normale Superiore in $2012,{ }^{26}$ which we thank for support and hospitality. The research of M.T. was partially supported by the European Union's Horizon 2020 research and innovation programme under the Marie Sklodowska-Curie grant agreement No 747228, by the program "Rita Levi Montalcini" of the MIUR (Minister for Instruction, University and Research) and by the INFN initiative STEFI. The work of E.J. was supported by National Research Foundation (Korea) through the grant NRF-2019R1F1A1044065.

\section{A YM \& Gravity examples}

The aim of this appendix is to give some examples of the general formalism and results which we have discussed in this note.

Focusing first on the YM case we shall start fom the cubic vertex

$$
\mathcal{C}_{123}^{(3)}=g\left(\partial_{u_{1}} \cdot \partial_{x_{23}} \partial_{u_{2}} \cdot \partial_{u_{3}}+\partial_{u_{2}} \cdot \partial_{x_{31}} \partial_{u_{3}} \cdot \partial_{u_{1}}+\partial_{u_{3}} \cdot \partial_{x_{12}} \partial_{u_{1}} \cdot \partial_{u_{2}}\right) \cdot
$$

For simplicity we already fixed the above color ordered form assuming that all coupling constants for different gauge fields are equal to $\mathrm{g}$. It is also possible to consider different coupling constants to start with and use the procedure outlined below to fix them from the requirement of locality (see e.g. $[1,7]$ )! In the following for convenience we shall fix $g=1$ without loss of generality. We can then easily evaluate the color ordered part of the exchange:

$$
\mathcal{E}_{1234}=\mathcal{C}_{12 u}^{(3)} \mathcal{C}_{34 v}^{(3)} \frac{u \cdot v}{\mathrm{~s}}+\mathcal{C}_{41 u}^{(3)} \mathcal{C}_{23 v}^{(3)} \frac{u \cdot v}{\mathrm{u}}
$$

\footnotetext{
${ }^{25}$ It was also argued in [21] that massless higher spins in AdS would not admit such a solution, although it is possible to fix the on-shell part of the homogeneous solution via holography [49, 63-65] or global higherspin symmetry $[21,48]$. The off-shell contribution would lead to divergences of the $1 / \square$ type associated to physical exchange contribution within the contact term, putting under question a purely field theoretic formulation of the theory.

${ }^{26}$ Some of these results were presented in the $2012 \mathrm{Ph} . \mathrm{D}$. thesis [2] by one of us.
} 
Considering the gauge variation with respect to leg 1 of the cubic structures one then gets:

$$
\delta_{\epsilon_{1}}^{(0)} \mathcal{C}_{12 u}^{(3)}=\left(\partial_{x_{2}}^{2}-\partial_{x_{u}}^{2}\right) \partial_{u_{2}} \cdot \partial_{u}-\partial_{u} \cdot\left(\partial_{x_{3}}+\partial_{x_{4}}\right) \partial_{u_{2}} \cdot\left(\partial_{x_{3}}+\partial_{x_{4}}\right)+\partial_{u} \cdot \partial_{x_{2}} \partial_{u_{2}} \cdot \partial_{x_{2}}
$$

Working off-shell with respect to the external leg but factoring out terms proportional to divergences and traces we can then write

$$
\delta_{\epsilon_{1}}^{(0)} \mathcal{C}_{12 u}^{(3)}=\left(\partial_{x_{2}}^{2}-\partial_{x_{u}}^{2}\right) \partial_{u_{2}} \cdot \partial_{u}-\partial_{u} \cdot\left(\partial_{x_{3}}+\partial_{x_{4}}\right) \partial_{u_{2}} \cdot\left(\partial_{x_{3}}+\partial_{x_{4}}\right)
$$

The term proportional to $\partial_{x_{u}}^{2}$ compensates the propagator pole in the exchange, generating a local gauge variation. On the other hand the term proportional to $\partial_{u} \cdot\left(\partial_{x_{3}}+\partial_{x_{4}}\right)$ computes the divergence of $\mathcal{V}_{34 v}$ which is a conserved current on-shell, therefore generating further terms proportional to $\partial_{x_{3}}^{2}$ and $\partial_{x_{4}}^{2}$ :

$$
\partial_{v} \cdot\left(\partial_{x_{3}}+\partial_{x_{4}}\right) \mathcal{C}_{34 v}^{(3)}=\left(\partial_{x_{3}}^{2}-\partial_{x_{4}}^{2}\right) \partial_{u_{3}} \cdot \partial_{u_{4}}+\partial_{u_{3}} \cdot \partial_{x_{4}} \partial_{u_{4}} \cdot \partial_{x_{4}}-\partial_{u_{4}} \cdot \partial_{x_{3}} \partial_{u_{3}} \cdot \partial_{x_{3}}
$$

It is interesting to notice that choosing a different representative for $\mathcal{V}_{12 u}$ like the cyclic ansatz:

$$
\mathcal{C}_{123}^{(3)}=g\left(\partial_{u_{1}} \cdot \partial_{x_{2}} \partial_{u_{2}} \cdot \partial_{u_{3}}+\partial_{u_{2}} \cdot \partial_{x_{3}} \partial_{u_{3}} \cdot \partial_{u_{1}}+\partial_{u_{3}} \cdot \partial_{x_{1}} \partial_{u_{1}} \cdot \partial_{u_{2}}\right)
$$

gives a gauge variation which precisely differ by such divergence terms. Consistency of the massless interaction ensures that such terms are proportional to the EoMs of the external legs. Gathering all such terms one obtains a rather cumbersome non-local deformation of the gauge transformations!

In the following we want to show that there exist a field and gauge parameter redefinition which removes the above non-local contributions to the gauge transformations. To this end, we proceed by solving for the homogeneous solution imposing locality of the quartic vertex on-shell. The most general ansatz for the homogeneous solution $\mathcal{C}_{1234}^{(4)}=$ $f\left(h_{i j}, h_{i}^{j k}, w^{i j}\right)$ reads in this case:

$$
\begin{aligned}
\mathcal{C}_{1234}^{(4)}= & c_{1} h_{14} h_{23}+c_{2} h_{13} h_{24}+c_{3} h_{12} h_{34} \\
& +c_{4} h_{1}^{34} h_{2}^{34} h_{34}+c_{5} h_{1}^{34} h_{24} h_{3}^{12}+c_{6} h_{14} h_{2}^{34} h_{3}^{12} \\
& +c_{7} h_{1}^{34} h_{23} h_{4}^{12}+c_{8} h_{13} h_{2}^{34} h_{4}^{12}+c_{9} h_{12} h_{3}^{12} h_{4}^{12} \\
& +c_{10} h_{1}^{34} h_{2}^{34} h_{3}^{12} h_{4}^{12},
\end{aligned}
$$

where we have fixed the on-shell redundancies in the $h$ monomials by picking the following ten independent structures:

$$
\begin{aligned}
& h_{12}, \quad h_{34}, \quad h_{13}, \quad h_{24}, \quad h_{14}, \quad h_{23} \text {, } \\
& h_{1}^{34}, \quad h_{2}^{34}, \quad h_{3}^{12}, \quad h_{4}^{12} .
\end{aligned}
$$

For instance only one among $h_{1}^{23}, h_{1}^{24}$ and $h_{1}^{34}$ is independent on-shell:

$$
h_{1}^{23}-h_{1}^{34} \approx 0, \quad h_{1}^{24}+h_{1}^{34} \approx 0 .
$$


In the ansatz (A.7) we have also assumed that the coefficients of each structure are (possibly non-local) functions of $w^{i j}$. The Noether procedure equation solves the quartic couplings as the following (generically non-local) functional:

$$
\mathcal{V}_{1234}=\mathcal{C}_{h 1234}^{(4)}-\mathcal{E}_{1234}
$$

Obviously, the Noether procedure equation above is empty unless further conditions on $\mathcal{V}_{1234}$ are enforced. Imposing locality of $\mathcal{V}_{1234}$ on-shell implies by dimensional analysis the following parameterisation for the quartic coupling:

$$
\mathcal{V}_{1234} \approx d_{1} \partial_{u_{1}} \cdot \partial_{u_{2}} \partial_{u_{3}} \cdot \partial_{u_{4}}+d_{2} \partial_{u_{1}} \cdot \partial_{u_{3}} \partial_{u_{2}} \cdot \partial_{u_{4}}+d_{3} \partial_{u_{1}} \cdot \partial_{u_{4}} \partial_{u_{2}} \cdot \partial_{u_{3}}
$$

where the equality $\approx$ holds on-shell with respect to the external legs. Note that by dimensional analysis a local vertex cannot be proportional in this case to $u_{i} \cdot p_{j}$. We can now solve (A.11). We do this by first setting $\partial_{x_{i}}^{2} \approx 0, \partial_{x_{i}} \cdot \partial_{u_{i}} \approx 0$ and $\mathrm{t} \approx-\mathrm{s}-\mathrm{u}$ and integrating by parts all $\partial_{x_{4}}$ derivatives. After eliminating all on-shell redundancies eq. (A.11) becomes a simple linear equation for the coefficients $c_{i}$ and $d_{i}$. Its solution can be shown to be unique and reads for the $d_{i}$ :

$$
d_{1}=-2, \quad d_{2}=4, \quad d_{3}=-2,
$$

reproducing the YM quartic vertex in its color ordered form. The coefficients $c_{i}$ then read:

$$
\begin{aligned}
& c_{1}=\frac{4 \mathrm{t}}{\mathrm{s}^{3}}, \quad c_{2}=\frac{4}{\mathrm{t}^{2}}, \quad c_{3}=\frac{4 \mathrm{t}}{\mathrm{u}^{3}} \quad c_{4}=c_{9}=\frac{4}{\mathrm{su}^{3}}, \\
& c_{6}=c_{7}=\frac{4}{\mathrm{u} \mathrm{s}^{3}}, \quad c_{5}=c_{8}=\frac{4}{\mathrm{sut}^{2}}, \quad c_{10}=-\frac{4\left(\mathrm{~s}^{2}+\mathrm{su}+\mathrm{u}^{2}\right)}{\mathrm{s}^{3} \mathrm{u}^{3} \mathrm{t}^{2}} .
\end{aligned}
$$

The above choice of coefficients ensures that the contact term $\mathcal{V}_{1234}$ is local on-shell! However off-shell the contact term $\mathcal{V}_{1234}=\mathcal{C}^{(4)}-\mathcal{E}_{1234}$ is actually non-local, which explains the non-local form of the deformations of the gauge transformations which can be obtained from the current exchange amplitude $\mathcal{E}_{1234}$, as discussed at the beginning of this section. However the fact that $\mathcal{V}_{1234}$ is local on-shell also implies that there exist a non-singular field redefinition which makes $\mathcal{V}_{1234}$ manifestly local. This implies that the non-local gauge deformations are in fact removable by a non-local non-singular field redefinition which maps off-shell $\mathcal{V}_{1234}$ to its manifestly local form (A.11). It is important to stress that the field redefinition that maps the two forms of the contact term is also non-local involving inverse of the Mandelstam invariants. This precisely accounts for the non-local deformation of the gauge transformation obtained from the exchange amplitude at the beginning of this section. The explicit form of the field redefinition is quite cumbersome and is not needed for our argument.

Similar but more cumbersome results can also be obtained in the case of massless spin-2 self-interactions. Skipping the details, after imposing locality on-shell of the solution 
$\mathcal{V}=\mathcal{C}^{(4)}-\mathcal{E}$ we find the following unique two-derivative quartic vertex:

$$
\begin{aligned}
& \mathcal{V}=16\left[-2\left(y_{3}^{1}\right)\left(y_{4}^{2}\right)\left(z_{12}\right)\left(z_{14}\right)\left(z_{23}\right)-\left(y_{3}^{2}\right)\left(y_{4}^{2}\right)\left(z_{12}\right)\left(z_{14}\right)\left(z_{23}\right)-\left(y_{3}^{2}\right)\left(y_{4}^{3}\right)\left(z_{12}\right)\left(z_{14}\right)\left(z_{23}\right)\right. \\
& -\left(y_{2}^{3}\right)\left(y_{4}^{2}\right)\left(z_{13}\right)\left(z_{14}\right)\left(z_{23}\right)-2\left(y_{2}^{1}\right)\left(y_{4}^{3}\right)\left(z_{13}\right)\left(z_{14}\right)\left(z_{23}\right)-\left(y_{2}^{3}\right)\left(y_{4}^{3}\right)\left(z_{13}\right)\left(z_{14}\right)\left(z_{23}\right) \\
& -2\left(y_{2}^{1}\right)\left(y_{3}^{1}\right)\left(z_{14}\right)^{2}\left(z_{23}\right)-\left(y_{2}^{3}\right)\left(y_{3}^{1}\right)\left(z_{14}\right)^{2}\left(z_{23}\right)-\left(y_{2}^{1}\right)\left(y_{3}^{2}\right)\left(z_{14}\right)^{2}\left(z_{23}\right) \\
& +\left(y_{1}^{3}\right)\left(y_{4}^{2}\right)\left(z_{14}\right)\left(z_{23}\right)^{2}+\left(y_{1}^{2}\right)\left(y_{4}^{3}\right)\left(z_{14}\right)\left(z_{23}\right)^{2}-\frac{\mathbf{u}}{4}\left(z_{14}\right)^{2}\left(z_{23}\right)^{2} \\
& +\left(y_{3}^{1}\right)\left(y_{4}^{2}\right)\left(z_{12}\right)\left(z_{13}\right)\left(z_{24}\right)+2\left(y_{3}^{2}\right)\left(y_{4}^{2}\right)\left(z_{12}\right)\left(z_{13}\right)\left(z_{24}\right)+2\left(y_{3}^{2}\right)\left(y_{4}^{3}\right)\left(z_{12}\right)\left(z_{13}\right)\left(z_{24}\right) \\
& -\left(y_{2}^{3}\right)\left(y_{4}^{2}\right)\left(z_{13}\right)^{2}\left(z_{24}\right)+\left(y_{2}^{1}\right)\left(y_{4}^{3}\right)\left(z_{13}\right)^{2}\left(z_{24}\right)-\left(y_{2}^{3}\right)\left(y_{4}^{3}\right)\left(z_{13}\right)^{2}\left(z_{24}\right) \\
& +\left(y_{2}^{1}\right)\left(y_{3}^{1}\right)\left(z_{13}\right)\left(z_{14}\right)\left(z_{24}\right)-\left(y_{2}^{3}\right)\left(y_{3}^{1}\right)\left(z_{13}\right)\left(z_{14}\right)\left(z_{24}\right)+2\left(y_{2}^{1}\right)\left(y_{3}^{2}\right)\left(z_{13}\right)\left(z_{14}\right)\left(z_{24}\right) \\
& +\left(y_{1}^{3}\right)\left(y_{4}^{2}\right)\left(z_{13}\right)\left(z_{23}\right)\left(z_{24}\right)-2\left(y_{1}^{2}\right)\left(y_{4}^{3}\right)\left(z_{13}\right)\left(z_{23}\right)\left(z_{24}\right)+2\left(y_{1}^{2}\right)\left(y_{3}^{1}\right)\left(z_{14}\right)\left(z_{23}\right)\left(z_{24}\right) \\
& +\left(y_{1}^{2}\right)\left(y_{3}^{2}\right)\left(z_{14}\right)\left(z_{23}\right)\left(z_{24}\right)-\left(y_{1}^{3}\right)\left(y_{3}^{2}\right)\left(z_{14}\right)\left(z_{23}\right)\left(z_{24}\right)-2 s\left(z_{13}\right)\left(z_{14}\right)\left(z_{23}\right)\left(z_{24}\right) \\
& \text { - }\left(y_{1}^{2}\right)\left(y_{3}^{1}\right)\left(z_{13}\right)\left(z_{24}\right)^{2}-2\left(y_{1}^{2}\right)\left(y_{3}^{2}\right)\left(z_{13}\right)\left(z_{24}\right)^{2}-\left(y_{1}^{3}\right)\left(y_{3}^{2}\right)\left(z_{13}\right)\left(z_{24}\right)^{2} \\
& -\frac{\mathrm{t}}{4}\left(z_{13}\right)^{2}\left(z_{24}\right)^{2}+\left(y_{3}^{1}\right)\left(y_{4}^{2}\right)\left(z_{12}\right)^{2}\left(z_{34}\right)-\left(y_{3}^{2}\right)\left(y_{4}^{2}\right)\left(z_{12}\right)^{2}\left(z_{34}\right) \\
& -\left(y_{3}^{2}\right)\left(y_{4}^{3}\right)\left(z_{12}\right)^{2}\left(z_{34}\right)+2\left(y_{2}^{3}\right)\left(y_{4}^{2}\right)\left(z_{12}\right)\left(z_{13}\right)\left(z_{34}\right)+\left(y_{2}^{1}\right)\left(y_{4}^{3}\right)\left(z_{12}\right)\left(z_{13}\right)\left(z_{34}\right) \\
& +2\left(y_{2}^{3}\right)\left(y_{4}^{3}\right)\left(z_{12}\right)\left(z_{13}\right)\left(z_{34}\right)+\left(y_{2}^{1}\right)\left(y_{3}^{1}\right)\left(z_{12}\right)\left(z_{14}\right)\left(z_{34}\right)+2\left(y_{2}^{3}\right)\left(y_{3}^{1}\right)\left(z_{12}\right)\left(z_{14}\right)\left(z_{34}\right) \\
& \text { - }\left(y_{2}^{1}\right)\left(y_{3}^{2}\right)\left(z_{12}\right)\left(z_{14}\right)\left(z_{34}\right)-2\left(y_{1}^{3}\right)\left(y_{4}^{2}\right)\left(z_{12}\right)\left(z_{23}\right)\left(z_{34}\right)+\left(y_{1}^{2}\right)\left(y_{4}^{3}\right)\left(z_{12}\right)\left(z_{23}\right)\left(z_{34}\right) \\
& +2\left(y_{1}^{3}\right)\left(y_{2}^{1}\right)\left(z_{14}\right)\left(z_{23}\right)\left(z_{34}\right)-\left(y_{1}^{2}\right)\left(y_{2}^{3}\right)\left(z_{14}\right)\left(z_{23}\right)\left(z_{34}\right)+\left(y_{1}^{3}\right)\left(y_{2}^{3}\right)\left(z_{14}\right)\left(z_{23}\right)\left(z_{34}\right) \\
& -2 \mathrm{t}\left(z_{12}\right)\left(z_{14}\right)\left(z_{23}\right)\left(z_{34}\right)-\left(y_{1}^{2}\right)\left(y_{3}^{1}\right)\left(z_{12}\right)\left(z_{24}\right)\left(z_{34}\right)+\left(y_{1}^{2}\right)\left(y_{3}^{2}\right)\left(z_{12}\right)\left(z_{24}\right)\left(z_{34}\right) \\
& +2\left(y_{1}^{3}\right)\left(y_{3}^{2}\right)\left(z_{12}\right)\left(z_{24}\right)\left(z_{34}\right)-\left(y_{1}^{3}\right)\left(y_{2}^{1}\right)\left(z_{13}\right)\left(z_{24}\right)\left(z_{34}\right)+2\left(y_{1}^{2}\right)\left(y_{2}^{3}\right)\left(z_{13}\right)\left(z_{24}\right)\left(z_{34}\right) \\
& +\left(y_{1}^{3}\right)\left(y_{2}^{3}\right)\left(z_{13}\right)\left(z_{24}\right)\left(z_{34}\right)-2 \mathrm{u}\left(z_{12}\right)\left(z_{13}\right)\left(z_{24}\right)\left(z_{34}\right)-\left(y_{1}^{3}\right)\left(y_{2}^{1}\right)\left(z_{12}\right)\left(z_{34}\right)^{2} \\
& \left.-\left(y_{1}^{2}\right)\left(y_{2}^{3}\right)\left(z_{12}\right)\left(z_{34}\right)^{2}-2\left(y_{1}^{3}\right)\left(y_{2}^{3}\right)\left(z_{12}\right)\left(z_{34}\right)^{2}-\frac{\mathbf{s}}{4}\left(z_{12}\right)^{2}\left(z_{34}\right)^{2}\right],
\end{aligned}
$$

together with the following homogeneous solution $\mathcal{C}^{(4)}$ :

$$
\begin{aligned}
\mathcal{C}^{(4)} & =-\frac{16 \mathrm{st} h_{14}^{2} h_{23}^{2}}{\mathrm{u}^{5}}-\frac{32 \mathrm{~s} h_{13} h_{14} h_{23} h_{24}}{t^{2} \mathrm{u}^{2}}-\frac{16 \mathrm{su} h_{13}^{2} h_{24}^{2}}{t^{5}}-\frac{32 \mathrm{t} h_{12} h_{14} h_{23} h_{34}}{\mathrm{~s}^{2} \mathrm{u}^{2}}-\frac{32 \mathrm{u} h_{12} h_{13} h_{24} h_{34}}{\mathrm{~s}^{2} \mathrm{t}^{2}} \\
& -\frac{32 h_{14}\left(h_{1}^{34}\right) h_{23}\left(h_{2}^{34}\right) h_{34}}{\mathrm{~s}^{2} \mathrm{u}^{3}}-\frac{32 h_{13}\left(h_{1}^{34}\right) h_{24}\left(h_{2}^{34}\right) h_{34}}{\mathrm{~s}^{2} \mathrm{t}^{3}}+\frac{16 \mathrm{u}(\mathrm{s}+\mathrm{u}) h_{12}^{2} h_{34}^{2}}{\mathrm{~s}^{5}}-\frac{32 h_{12}\left(h_{1}^{34}\right)\left(h_{2}^{34}\right) h_{34}^{2}}{\mathrm{~s}^{5}} \\
& -\frac{16\left(h_{1}^{34}\right)^{2}\left(h_{2}^{34}\right)^{2} h_{34}^{2}}{\mathrm{~s}^{5} \mathrm{tu}}+\frac{32 h_{14}\left(h_{1}^{34}\right) h_{23} h_{24}\left(h_{3}^{12}\right)}{t^{2} \mathrm{u}^{3}}+\frac{32 h_{13}\left(h_{1}^{34}\right) h_{24}^{2}\left(h_{3}^{12}\right)}{t^{5}}-\frac{32 h_{14}^{2} h_{23}\left(h_{2}^{34}\right)\left(h_{3}^{12}\right)}{\mathrm{u}^{5}} \\
& -\frac{32 h_{13} h_{14} h_{24}\left(h_{2}^{34}\right)\left(h_{3}^{12}\right)}{t^{3} \mathrm{u}^{2}}+\frac{32 h_{12}\left(h_{1}^{34}\right) h_{24} h_{34}\left(h_{3}^{12}\right)}{\mathrm{s}^{3} \mathrm{t}^{2}}-\frac{32 h_{12} h_{14}\left(h_{2}^{34}\right) h_{34}\left(h_{3}^{12}\right)}{\mathrm{s}^{3} \mathrm{u}^{2}} \\
& +\frac{32\left(h_{1}^{34}\right)^{2} h_{24}\left(h_{2}^{34}\right) h_{34}\left(h_{3}^{12}\right)}{\mathrm{s}^{3} \mathrm{t}^{3} \mathrm{u}}-\frac{32 h_{14}\left(h_{1}^{34}\right)\left(h_{2}^{34}\right)^{2} h_{34}\left(h_{3}^{12}\right)}{\mathrm{s}^{3} \mathrm{tu}^{3}}-\frac{16\left(h_{1}^{34}\right)^{2} h_{24}^{2}\left(h_{3}^{12}\right)^{2}}{\mathrm{~s}^{5} \mathrm{u}} \\
& +\frac{32 h_{14}\left(h_{1}^{34}\right) h_{24}\left(h_{2}^{34}\right)\left(h_{3}^{12}\right)^{2}}{\mathrm{st}^{3} \mathrm{u}^{3}}-\frac{16 h_{14}^{2}\left(h_{2}^{34}\right)^{2}\left(h_{3}^{12}\right)^{2}}{\mathrm{stu}^{5}}-\frac{32 h_{14}\left(h_{1}^{34}\right) h_{23}^{2}\left(h_{4}^{12}\right)}{\mathrm{u}^{5}} \\
& -\frac{32 h_{13}\left(h_{1}^{34}\right) h_{23} h_{24}\left(h_{4}^{12}\right)}{t^{3} \mathrm{u}^{2}}+\frac{32 h_{13} h_{14} h_{23}\left(h_{2}^{34}\right)\left(h_{4}^{12}\right)}{t^{2} \mathrm{u}^{3}}+\frac{32 h_{13}^{2} h_{24}\left(h_{2}^{34}\right)\left(h_{4}^{12}\right)}{t^{5}}-\frac{32 h_{12}\left(h_{1}^{34}\right) h_{23} h_{34}\left(h_{4}^{12}\right)}{\mathrm{s}^{3} \mathrm{u}^{2}} \\
& +\frac{32 h_{12} h_{13}\left(h_{2}^{34}\right) h_{34}\left(h_{4}^{12}\right)}{\mathrm{s}^{3} \mathrm{t}^{2}}-\frac{32\left(h_{1}^{34}\right)^{2} h_{23}\left(h_{2}^{34}\right) h_{34}\left(h_{4}^{12}\right)}{\mathrm{s}^{3} \mathrm{tu}^{3}}+\frac{32 h_{13}\left(h_{1}^{34}\right)\left(h_{2}^{34}\right)^{2} h_{34}\left(h_{4}^{12}\right)}{\mathrm{s}^{3} \mathrm{t}^{3} \mathrm{u}}
\end{aligned}
$$




$$
\begin{aligned}
& -\frac{32 h_{12} h_{14} h_{23}\left(h_{3}^{12}\right)\left(h_{4}^{12}\right)}{\mathrm{s}^{2} \mathrm{u}^{3}}-\frac{32 h_{12} h_{13} h_{24}\left(h_{3}^{12}\right)\left(h_{4}^{12}\right)}{\mathrm{s}^{2} \mathrm{t}^{3}}+\frac{32\left(h_{1}^{34}\right)^{2} h_{23} h_{24}\left(h_{3}^{12}\right)\left(h_{4}^{12}\right)}{\mathrm{st}^{3} \mathrm{u}^{3}} \\
& +\frac{32\left(2 \mathrm{~s}^{2}+2 \mathrm{su}+\mathrm{u}^{2}\right) h_{14}\left(h_{1}^{34}\right) h_{23}\left(h_{2}^{34}\right)\left(h_{3}^{12}\right)\left(h_{4}^{12}\right)}{\mathrm{s}^{2} \mathbf{t}^{2} \mathbf{u}^{5}}+\frac{32\left(\mathrm{~s}^{2}+\mathrm{u}^{2}\right) h_{13}\left(h_{1}^{34}\right) h_{24}\left(h_{2}^{34}\right)\left(h_{3}^{12}\right)\left(h_{4}^{12}\right)}{\mathrm{s}^{2} \mathbf{t}^{5} \mathbf{u}^{2}} \\
& +\frac{32 h_{13} h_{14}\left(h_{2}^{34}\right)^{2}\left(h_{3}^{12}\right)\left(h_{4}^{12}\right)}{\mathrm{st}^{3} \mathrm{u}^{3}}-\frac{32 h_{12}^{2} h_{34}\left(h_{3}^{12}\right)\left(h_{4}^{12}\right)}{\mathrm{s}^{5}}+\frac{32\left(\mathrm{~s}^{2}+2 \mathrm{su}+2 \mathrm{u}^{2}\right) h_{12}\left(h_{1}^{34}\right)\left(h_{2}^{34}\right) h_{34}\left(h_{3}^{12}\right)\left(h_{4}^{12}\right)}{\mathrm{s}^{5} \mathrm{t}^{2} \mathrm{u}^{2}} \\
& +\frac{32\left(\mathrm{~s}^{2}+\mathrm{su}+\mathrm{u}^{2}\right)\left(h_{1}^{34}\right)^{2}\left(h_{2}^{34}\right)^{2} h_{34}\left(h_{3}^{12}\right)\left(h_{4}^{12}\right)}{\mathrm{s}^{5} \mathrm{t}^{3} \mathrm{u}^{3}}+\frac{32 h_{12}\left(h_{1}^{34}\right) h_{24}\left(h_{3}^{12}\right)^{2}\left(h_{4}^{12}\right)}{\mathrm{s}^{3} \mathrm{t}^{3} \mathrm{u}} \\
& -\frac{32 h_{12} h_{14}\left(h_{2}^{34}\right)\left(h_{3}^{12}\right)^{2}\left(h_{4}^{12}\right)}{\mathrm{s}^{3} \mathrm{tu}}-\frac{32\left(\mathrm{~s}^{2}+\mathrm{su}+\mathrm{u}^{2}\right)\left(h_{1}^{34}\right)^{2} h_{24}\left(h_{2}^{34}\right)\left(h_{3}^{12}\right)^{2}\left(h_{4}^{12}\right)}{\mathrm{s}^{3} \mathrm{t}^{5} \mathbf{u}^{3}} \\
& +\frac{32\left(\mathrm{~s}^{2}+\mathrm{su}+\mathrm{u}^{2}\right) h_{14}\left(h_{1}^{34}\right)\left(h_{2}^{34}\right)^{2}\left(h_{3}^{12}\right)^{2}\left(h_{4}^{12}\right)}{\mathrm{s}^{3} \mathrm{t}^{3} \mathrm{u}^{5}} \\
& -\frac{16\left(h_{1}^{34}\right)^{2} h_{23}^{2}\left(h_{4}^{12}\right)^{2}}{\mathrm{stu}^{5}}+\frac{32 h_{13}\left(h_{1}^{34}\right) h_{23}\left(h_{2}^{34}\right)\left(h_{4}^{12}\right)^{2}}{\mathrm{st}^{3} \mathbf{u}^{3}}-\frac{16 h_{13}^{2}\left(h_{2}^{34}\right)^{2}\left(h_{4}^{12}\right)^{2}}{\mathrm{st}^{5} \mathrm{u}} \\
& -\frac{32 h_{12}\left(h_{1}^{34}\right) h_{23}\left(h_{3}^{12}\right)\left(h_{4}^{12}\right)^{2}}{\mathbf{s}^{3} \mathrm{tu}^{3}}+\frac{32 h_{12} h_{13}\left(h_{2}^{34}\right)\left(h_{3}^{12}\right)\left(h_{4}^{12}\right)^{2}}{\mathrm{~s}^{3} \mathrm{t}^{3} \mathrm{u}}+\frac{32\left(\mathrm{~s}^{2}+\mathrm{su}+\mathrm{u}^{2}\right)\left(h_{1}^{34}\right)^{2} h_{23}\left(h_{2}^{34}\right)\left(h_{3}^{12}\right)\left(h_{4}^{12}\right)^{2}}{\mathrm{~s}^{3} \mathrm{t}^{3} \mathbf{u}^{5}} \\
& -\frac{32\left(\mathrm{~s}^{2}+\mathrm{su}+\mathrm{u}^{2}\right) h_{13}\left(h_{1}^{34}\right)\left(h_{2}^{34}\right)^{2}\left(h_{3}^{12}\right)\left(h_{4}^{12}\right)^{2}}{\mathrm{~s}^{3} \mathrm{t}^{5} \mathrm{u}^{3}}-\frac{16 h_{12}^{2}\left(h_{3}^{12}\right)^{2}\left(h_{4}^{12}\right)^{2}}{\mathrm{~s}^{5} \mathrm{tu}} \\
& +\frac{32\left(\mathrm{~s}^{2}+\mathrm{su}+\mathrm{u}^{2}\right) h_{12}\left(h_{1}^{34}\right)\left(h_{2}^{34}\right)\left(h_{3}^{12}\right)^{2}\left(h_{4}^{12}\right)^{2}}{\mathrm{~s}^{5} \mathrm{t}^{3} \mathrm{u}^{3}}-\frac{16\left(\mathrm{~s}^{2}+\mathrm{su}+\mathrm{u}^{2}\right)^{2}\left(h_{1}^{34}\right)^{2}\left(h_{2}^{34}\right)^{2}\left(h_{3}^{12}\right)^{2}\left(h_{4}^{12}\right)^{2}}{\mathrm{~s}^{5} \mathrm{t}^{5} \mathrm{u}^{5}} \text {. }
\end{aligned}
$$

Again, the price to pay for manifest gauge invariance is that locality and factorisation are not manifest.

In conclusion, our formalism can be easily implemented within a computer algebra program and given a set of exchange amplitudes allows to quickly find (if it exists) the corresponding contact term and as well the manifestly gauge invariant form of the homogeneous solution! With our formalism it is for instance straightforward to rule out local spin-3 self-interactions as well as interaction of a spin-3 field with any spectrum of higher-spin fields if a coupling of spin-3 and a massless spin-2 is non-vanishing.

Open Access. This article is distributed under the terms of the Creative Commons Attribution License (CC-BY 4.0), which permits any use, distribution and reproduction in any medium, provided the original author(s) and source are credited.

\section{References}

[1] M. Taronna, Higher-Spin Interactions: four-point functions and beyond, JHEP 04 (2012) 029 [arXiv: 1107.5843] [INSPIRE].

[2] M. Taronna, Higher-Spin Interactions: three-point functions and beyond, arXiv:1209.5755 [INSPIRE].

[3] D. Polyakov, Higher Spins and Open Strings: Quartic Interactions, Phys. Rev. D 83 (2011) 046005 [arXiv: 1011.0353 ] [INSPIRE].

[4] P. Dempster and M. Tsulaia, On the Structure of Quartic Vertices for Massless Higher Spin Fields on Minkowski Background, Nucl. Phys. B 865 (2012) 353 [arXiv:1203.5597] [INSPIRE].

[5] A.K.H. Bengtsson, Investigations into Light-front Quartic Interactions for Massless Fields (I): Non-constructibility of Higher Spin Quartic Amplitudes, JHEP 12 (2016) 134 [arXiv: 1607.06659] [INSPIRE]. 
[6] D. Ponomarev, Off-Shell Spinor-Helicity Amplitudes from Light-Cone Deformation Procedure, JHEP 12 (2016) 117 [arXiv:1611.00361] [INSPIRE].

[7] M. Taronna, On the Non-Local Obstruction to Interacting Higher Spins in Flat Space, JHEP 05 (2017) 026 [arXiv: 1701.05772] [INSPIRE].

[8] R. Roiban and A.A. Tseytlin, On four-point interactions in massless higher spin theory in flat space, JHEP 04 (2017) 139 [arXiv:1701.05773] [INSPIRE].

[9] D. Polyakov and C. Zhang, Higher-Spin Quartic Vertices in AdS $S_{5}$ : a Rectangular Limit, arXiv:1809.10580 [INSPIRE].

[10] F.A. Berends, G.J.H. Burgers and H. van Dam, On the Theoretical Problems in Constructing Interactions Involving Higher Spin Massless Particles, Nucl. Phys. B 260 (1985) 295 [INSPIRE].

[11] F.A. Berends, J.W. van Holten, B. de Wit and P. van Nieuwenhuizen, On Spin 5/2 Gauge Fields, J. Phys. A 13 (1980) 1643 [inSPIRE].

[12] F.A. Berends, G.J.H. Burgers and H. Van Dam, On spin three selfinteractions, Z. Phys. C 24 (1984) 247 [INSPIRE].

[13] G. Barnich and M. Henneaux, Consistent couplings between fields with a gauge freedom and deformations of the master equation, Phys. Lett. B 311 (1993) 123 [hep-th/9304057] [INSPIRE].

[14] R.R. Metsaev, Cubic interaction vertices of massive and massless higher spin fields, Nucl. Phys. B 759 (2006) 147 [hep-th/0512342] [INSPIRE].

[15] N. Boulanger, S. Leclercq and P. Sundell, On The Uniqueness of Minimal Coupling in Higher-Spin Gauge Theory, JHEP 08 (2008) 056 [arXiv: 0805. 2764] [INSPIRE].

[16] X. Bekaert, N. Boulanger and S. Leclercq, Strong obstruction of the Berends-Burgers-van Dam spin-3 vertex, J. Phys. A 43 (2010) 185401 [arXiv:1002.0289] [InSPIRE].

[17] R. Manvelyan, K. Mkrtchyan and W. Rühl, General trilinear interaction for arbitrary even higher spin gauge fields, Nucl. Phys. B 836 (2010) 204 [arXiv: 1003.2877] [InSPIRE].

[18] A. Sagnotti and M. Taronna, String Lessons for Higher-Spin Interactions, Nucl. Phys. B 842 (2011) 299 [arXiv: 1006.5242] [INSPIRE].

[19] E. Joung and M. Taronna, Cubic interactions of massless higher spins in (A)dS: metric-like approach, Nucl. Phys. B 861 (2012) 145 [arXiv:1110.5918] [InSPIRE].

[20] E. Joung, L. Lopez and M. Taronna, Solving the Noether procedure for cubic interactions of higher spins in (A)dS, J. Phys. A 46 (2013) 214020 [arXiv:1207.5520] [INSPIRE].

[21] C. Sleight and M. Taronna, Higher-Spin Gauge Theories and Bulk Locality, Phys. Rev. Lett. 121 (2018) 171604 [arXiv:1704.07859] [INSPIRE].

[22] P. Kessel, G. Lucena Gómez, E. Skvortsov and M. Taronna, Higher Spins and Matter Interacting in Dimension Three, JHEP 11 (2015) 104 [arXiv:1505.05887] [INSPIRE].

[23] E.D. Skvortsov and M. Taronna, On Locality, Holography and Unfolding, JHEP 11 (2015) 044 [arXiv: 1508.04764] [INSPIRE].

[24] M. Taronna, Pseudo-local Theories: A Functional Class Proposal, in International Workshop on Higher Spin Gauge Theories, pp. 59-84, 2017, DOI [arXiv:1602.08566] [INSPIRE]. 
[25] M. Taronna, A note on field redefinitions and higher-spin equations, J. Phys. A 50 (2017) 075401 [arXiv: 1607.04718] [INSPIRE].

[26] V.E. Didenko, O.A. Gelfond, A.V. Korybut and M.A. Vasiliev, Limiting Shifted Homotopy in Higher-Spin Theory and Spin-Locality, JHEP 12 (2019) 086 [arXiv:1909.04876] [INSPIRE].

[27] M.A. Vasiliev, Consistent equation for interacting gauge fields of all spins in (3+1)-dimensions, Phys. Lett. B 243 (1990) 378 [inSPIRE].

[28] J. Engquist and P. Sundell, Brane partons and singleton strings, Nucl. Phys. B 752 (2006) 206 [hep-th/0508124] [INSPIRE].

[29] N. Boulanger and P. Sundell, An action principle for Vasiliev's four-dimensional higher-spin gravity, J. Phys. A 44 (2011) 495402 [arXiv:1102.2219] [INSPIRE].

[30] N. Boulanger, N. Colombo and P. Sundell, A minimal BV action for Vasiliev's four-dimensional higher spin gravity, JHEP 10 (2012) 043 [arXiv:1205.3339] [INSPIRE].

[31] R. Bonezzi, N. Boulanger, E. Sezgin and P. Sundell, Frobenius-Chern-Simons gauge theory, J. Phys. A 50 (2017) 055401 [arXiv: 1607.00726] [InSPIRE].

[32] R. Bonezzi, N. Boulanger, D. De Filippi and P. Sundell, Noncommutative Wilson lines in higher-spin theory and correlation functions of conserved currents for free conformal fields, J. Phys. A 50 (2017) 475401 [arXiv: 1705.03928] [INSPIRE].

[33] C. Iazeolla, E. Sezgin and P. Sundell, On Exact Solutions and Perturbative Schemes in Higher Spin Theory, Universe 4 (2018) 5 [arXiv:1711.03550] [INSPIRE].

[34] D. De Filippi, C. Iazeolla and P. Sundell, Fronsdal fields from gauge functions in Vasiliev's higher spin gravity, JHEP 10 (2019) 215 [arXiv:1905.06325] [INSPIRE].

[35] C. Sleight and M. Taronna, Spinning Witten Diagrams, JHEP 06 (2017) 100 [arXiv: 1702.08619] [INSPIRE].

[36] M.P. Blencowe, A Consistent Interacting Massless Higher Spin Field Theory in D = (2+1), Class. Quant. Grav. 6 (1989) 443 [InSPIRE].

[37] M. Henneaux and S.-J. Rey, Nonlinear $W_{\infty}$ as Asymptotic Symmetry of Three-Dimensional Higher Spin Anti-de Sitter Gravity, JHEP 12 (2010) 007 [arXiv: 1008.4579] [INSPIRE].

[38] A. Campoleoni, S. Fredenhagen, S. Pfenninger and S. Theisen, Asymptotic symmetries of three-dimensional gravity coupled to higher-spin fields, JHEP 11 (2010) 007 [arXiv: 1008.4744] [INSPIRE].

[39] A.A. Tseytlin, On limits of superstring in $A d S_{5} \times S^{5}$, Theor. Math. Phys. 133 (2002) 1376 [hep-th/0201112] [INSPIRE].

[40] A.Y. Segal, Conformal higher spin theory, Nucl. Phys. B 664 (2003) 59 [hep-th/0207212] [INSPIRE].

[41] X. Bekaert, E. Joung and J. Mourad, Effective action in a higher-spin background, JHEP 02 (2011) 048 [arXiv: 1012.2103] [INSPIRE].

[42] R.R. Metsaev, Poincaré invariant dynamics of massless higher spins: Fourth order analysis on mass shell, Mod. Phys. Lett. A 6 (1991) 359 [InSPIRE].

[43] R.R. Metsaev, S matrix approach to massless higher spins theory. 2: The case of internal symmetry, Mod. Phys. Lett. A 6 (1991) 2411 [INSPIRE]. 
[44] R. Metsaev, Effective Action in String Theory, Ph.D. Thesis, Lebedev Physical Institute, Moscow, Russia, (1991).

[45] D. Ponomarev and E.D. Skvortsov, Light-Front Higher-Spin Theories in Flat Space, J. Phys. A 50 (2017) 095401 [arXiv: 1609.04655] [INSPIRE].

[46] E. Joung and M. Taronna, Cubic-interaction-induced deformations of higher-spin symmetries, JHEP 03 (2014) 103 [arXiv:1311.0242] [INSPIRE].

[47] N. Boulanger, D. Ponomarev, E.D. Skvortsov and M. Taronna, On the uniqueness of higher-spin symmetries in AdS and CFT, Int. J. Mod. Phys. A 28 (2013) 1350162 [arXiv: 1305.5180] [INSPIRE].

[48] C. Sleight and M. Taronna, Higher-Spin Algebras, Holography and Flat Space, JHEP 02 (2017) 095 [arXiv : 1609.00991] [INSPIRE].

[49] C. Sleight and M. Taronna, Higher Spin Interactions from Conformal Field Theory: The Complete Cubic Couplings, Phys. Rev. Lett. 116 (2016) 181602 [arXiv:1603.00022] [INSPIRE].

[50] C. Sleight and M. Taronna, Feynman rules for higher-spin gauge fields on AdS $S_{d+1}$, JHEP 01 (2018) 060 [arXiv: 1708.08668] [INSPIRE].

[51] E. Joung, L. Lopez and M. Taronna, On the cubic interactions of massive and partially-massless higher spins in (A)dS, JHEP 07 (2012) 041 [arXiv:1203.6578] [INSPIRE].

[52] E. Joung, L. Lopez and M. Taronna, Generating functions of (partially-)massless higher-spin cubic interactions, JHEP 01 (2013) 168 [arXiv:1211.5912] [INSPIRE].

[53] X. Bekaert, N. Boulanger and S. Cnockaert, Spin three gauge theory revisited, JHEP 01 (2006) 052 [hep-th/0508048] [INSPIRE].

[54] T. Damour and S. Deser, Higher Derivative Interactions of Higher Spin Gauge Fields, Class. Quant. Grav. 4 (1987) L95 [INSPIRE].

[55] A. Campoleoni, S. Fredenhagen, S. Pfenninger and S. Theisen, Towards metric-like higher-spin gauge theories in three dimensions, J. Phys. A 46 (2013) 214017 [arXiv:1208.1851] [INSPIRE].

[56] K. Mkrtchyan, Cubic interactions of massless bosonic fields in three dimensions, Phys. Rev. Lett. 120 (2018) 221601 [arXiv: 1712.10003] [INSPIRE].

[57] P. Kessel and K. Mkrtchyan, Cubic interactions of massless bosonic fields in three dimensions II: Parity-odd and Chern-Simons vertices, Phys. Rev. D 97 (2018) 106021 [arXiv: 1803.02737] [INSPIRE].

[58] S. Fredenhagen, O. Krüger and K. Mkrtchyan, Constraints for Three-Dimensional Higher-Spin Interactions and Conformal Correlators, Phys. Rev. D 100 (2019) 066019 [arXiv: 1812.10462] [INSPIRE].

[59] S. Fredenhagen, O. Krüger and K. Mkrtchyan, Vertex-Constraints in 3D Higher Spin Theories, Phys. Rev. Lett. 123 (2019) 131601 [arXiv: 1905. 00093] [InSPIRE].

[60] R. Manvelyan, R. Mkrtchyan and W. Ruehl, Radial Reduction and Cubic Interaction for Higher Spins in (A)dS space, Nucl. Phys. B 872 (2013) 265 [arXiv:1210.7227] [inSPIRE].

[61] D. Francia, G.L. Monaco and K. Mkrtchyan, Cubic interactions of Maxwell-like higher spins, JHEP 04 (2017) 068 [arXiv: 1611.00292] [INSPIRE]. 
[62] M. Karapetyan, R. Manvelyan and R. Poghossian, Cubic interaction for higher spins in $A d S_{d+1}$ space in the explicit covariant form, Nucl. Phys. B 950 (2020) 114876 [arXiv: 1908.07901] [INSPIRE].

[63] X. Bekaert, J. Erdmenger, D. Ponomarev and C. Sleight, Towards holographic higher-spin interactions: Four-point functions and higher-spin exchange, JHEP 03 (2015) 170 [arXiv: 1412.0016] [INSPIRE].

[64] X. Bekaert, J. Erdmenger, D. Ponomarev and C. Sleight, Quartic AdS Interactions in Higher-Spin Gravity from Conformal Field Theory, JHEP 11 (2015) 149 [arXiv: 1508. 04292] [INSPIRE].

[65] C. Sleight, Interactions in higher-spin gravity: a holographic perspective, J. Phys. A50 (2017) 383001 [arXiv: 1610.01318] [INSPIRE]. 